\title{
Efficient NFV-Enabled Multicasting in SDNs
}

\author{
Zichuan Xu, Member, IEEE, Weifa Liang, Senior Member, IEEE, Meitian Huang, Mike Jia, Song \\ Guo, Senior Member, IEEE, and Alex Galis, Member, IEEE
}

\begin{abstract}
Multicasting is a fundamental functionality of many network applications, including online conferencing, event monitoring, video streaming, and so on. To ensure reliable, secure and scalable multicasting, a service chain that consists of network functions (e.g., firewalls, Intrusion Detection Systems (IDSs), and transcoders) usually is associated with each multicast request. We refer to such a multicast request with service chain requirement as an NFV-enabled multicast request. In this paper, we study NFV-enabled multicasting in a SoftwareDefined Network (SDN) with an aim to maximize network throughput while minimizing the implementation cost of admitted NFV-enabled multicast requests, subject to network resource capacity, where the implementation cost of a request consists of its computing resource consumption cost in servers and its network bandwidth consumption cost when routing and processing its data packets in the network. To this end, we first formulate two NFV-enabled multicasting problems with and without resource capacity constraints and one online NFV-enabled multicasting problem. We then devise two approximation algorithms with an approximation ratio of $2 M$ for the NFV-enabled multicasting problems with and without resource capacity constraints, if the number of servers for implementing the service chain of each request is no greater than a constant $M(\geq 1)$. We also study dynamic admissions of NFV-enabled multicast requests without the knowledge of future request arrivals with the objective to maximize the network throughput, for which we propose an efficient heuristic, and for a special case of dynamic request admissions, we devise an online algorithm with a competitive ratio of $O(\log n)$ for it when $M=1$, where $n$ is the number of nodes in the network. We finally evaluate the performance of the proposed algorithms through experimental simulations. Experimental results demonstrate that the proposed algorithms are promising and outperform existing heuristics.
\end{abstract}

Index Terms-Network function virtualization; software-defined networks; multicasting; NFV-enabled multicasting; service chains; virtualized network functions; routing; approximation and online algorithms.

\section{INTRODUCTION}

Today's data centers and communication networks deploy a variety of intermediary middleboxes, e.g., firewalls, Intrusion Detection Systems (IDSs), proxies, and WAN optimizers, to guarantee the security and performance of

- Z. Xu is with the School of Software, Dalian University of Technology, and the Key Laboratory for Ubiquitous Network and Service Software of Liaoning Province, China, 116621. E-mails: z.xu@dlut.edu.cn

- W. Liang, M. Huang, and M. Jia are with the Research School of Computer Science, the Australian National University, Canberra, ACT 2601, Australia.E-mails: wliang@cs.anu.edu.au, meitian.huang@anu.edu.au, and u5515287@anu.edu.au

- S. Guo is with the Department of Computing, the Hong Kong Polytechnic University, Hong Kong. E-mail: song.guo@polyu.edu.hk

- A. Galis is with the Department of Electronic E Electrical Engineering, University College London, London WC1E 7JE, UK. E-mails: a.galis@ucl.ac.uk data transfers. However, considering that the middleboxes are typically made by expensive dedicated hardware and managed manually, they not only raise the capital expenditures (CapEx) and operating expenses (OpEx) of many service providers but also increase the inflexibility of their management dramatically. Network Function Virtualization (NFV) [6], [7], [12], [27] has been emerging as a promising paradigm to reduce the CapEx and OpEx of service providers, by implementing network functions as software components running in Virtual Machines (VMs). Underpinned by the NFV technique, Software-Defined Networking (SDN) can be further utilized to enable flexible implementations of network functions, by leveraging flexible control and management empowered by the separation of the control plane and the data plane.

Multicasting is a widely-used type of communication in the mentioned NFV-enabled SDNs. Each multicast request transmits data from one source to multiple destinations. The applications of multicasting include video conferencing, multimedia distribution, and software updates in data centers. In data center networks, multicasting is widely adopted by many applications from front-end delay-sensitive cloud applications, to back-end bandwidth-hungry computations [30]. Examples include directing search queries to a set of indexing servers [3], distributing executable binaries to a group of servers participating cooperative computations such as MapReduce [28], upgrading OS and software on data center servers, replicating file chunks in distributing file systems [9]. All of such applications need network functions to guarantee the security or performance of their traffic. For example, an Application Delivery Controller (ADC) may be needed to replicate the data of an application to a group of servers, to meet the security needs of the application and provide simplified authentication, authorization and accounting [1]. In particular, with the SDN technology being envisioned as the dominant technology for the next-generation data center networks [36], [37], enabling efficient NFV-enabled multicasting in SDNs becomes an urgent task. Techniques designed for multicasting in conventional networks cannot be applied to NFV-enabled multicasting as they do not consider the placement of virtualized network functions (VNFs). In this paper, we thus aim to enable efficient NFV-enabled multicasting in SDNs via efficient and effective placement of VNFs for multicast requests that require forwarding their traffic to some specified middleboxes before reaching their destinations. To admit multicast requests with network function requirements that will be implemented in servers as VMs, we study the problem of NFV-enabled multicasting 
in a software-defined network that is equipped with servers to run the VMs.

Performing NFV-enabled multicasting in an SDN is significantly challenging. The VMs in servers for network function implementations are located at different locations of the SDN, and this poses a great challenge to minimize the cost of implementing multicast requests. Naive placement of the VMs of each NFV-enabled multicast request at locations that are far away from the source and/or the destinations of the request may incur a prohibitive communication cost. In addition, multicast requests usually arrive in the network one by one without the knowledge of future arrivals. This leads to difficulties to estimate dynamic workloads of both computing and bandwidth resources at servers and links. The challenges thus are (1) how to jointly find one or multiple servers to implement its network functions by finding a multicast tree for each multicast request while meeting its computing and bandwidth demands; (2) how to design a novel metric that can accurately capture the dynamic resource usages and workloads in the SDN; and (3) how to devise an online algorithm with a competitive ratio to maximize the number of multicast requests admitted, subject to resource capacity constraints.

Several studies on multicasting in SDNs have been conducted recently [15], [16], [42], [43]. However, most of them did not consider network functions in multicast requests [15], [16] or dealt with a sequence of requests without future arrival knowledge [42], [43]. In contrast, we here investigate NFV-enabled multicasting, by devising an approximation algorithm with a provable approximation ratio for realizing a single NFV-enabled multicast request. We also develop a first online algorithm with a guaranteed competitive ratio for the online NFV-enabled multicasting problem. To the best of our knowledge, we are the first to formulate a novel NFV-enabled multicast problem in SDNs with the aim to minimize its implementation cost, through striving for fine trade-offs between computing and bandwidth resource consumptions if no more than $M$ servers are employed to implement the service chain of each request. We devise the first approximation algorithm for the problem. We also study online NFV-enabled multicasting and devise the first online algorithm with a provable competitive ratio if only one server is deployed for its service chain implementation. The key ingredients in the design of both approximation and online algorithms are a series of non-trivial reductions. The developed analytical techniques for approximation and competitive ratios of the proposed algorithms may have independent of interest on the analysis of online algorithms for other optimization problems that involve different types of resource optimization.

The main contributions of this paper are as follows. We first study the problem of NFV-enabled multicasting in an SDN to minimize the implementation cost of each NFV-enabled multicast request, in terms of both computing and bandwidth resource consumptions. We then devise the first approximation algorithm with an approximation ratio of $2 M$ to minimize the implementation cost of each request, assuming that the number of servers used for implementing its service chain is no more than $M$. We also investigate dynamic admissions of NFV-enabled multicast requests without the knowledge of their future arrivals with an aim to maximize the network throughput by proposing an online algorithm. Furthermore, we devise a novel online algorithm with a provable competitive ratio for a special case of dynamic request admissions when $M=1$. We finally evaluate the performance of the proposed algorithms by experimental simulations. Experimental results show that the proposed algorithms outperform existing heuristics.

The rest of the paper is organized as follows. Section 2 reviews the related work. Section 3 introduces the system model, notations, and problem definitions. Section 4 devises approximation algorithms for the NFV-enabled multicasting problem with and without network resource capacity constraints. Section 5 devises online algorithms for the online NFV-enabled multicasting problem. Section 6 evaluates the performance of the proposed algorithms by experimental simulation, and Section 7 concludes the paper.

\section{Related Work}

SDN and NFV as emerging technologies have been shaping the future networking landscape, by bringing the promise of enabling inexpensive and flexible management solutions [18], [14], [34]. Traditional routing algorithms that are designed for conventional networks are inapplicable to NFV-enabled SDNs. Novel routing algorithms that jointly performs traffic routing and NFV placements are needed. There are studies that explored the issues on placement and resource allocation for NFVs in SDNs [6], [7], [22], [26], [31], [41]. Most of these studies however do not consider multicasting in SDNs. For example, Moens et al. [31] investigated efficient NFV placement in SDNs. They focused on a hybrid scenario where some network functions are implemented by dedicated physical hardware while others are implemented in VMs. Lukovszki et al. [26] studied the problem of online admission and embedding of service chains (i.e., a sequence of virtualized network functions) into a substrate network (i.e., an SDN with both bandwidth and computing resource capacities on its links and nodes, assuming that servers are installed at each node in the network. Li et al. [22] designed and implemented a system that enables dynamic resource provision in an NFV-enabled SDN. They also studied the problem of maximizing the total number of unicast requests that can be assigned to each service chain, by formulating an Integer Linear Programming (ILP) solution and developing a randomized rounding method. Cao et al. [5] considered policy-aware traffic engineering in SDNs, by assuming that the traffic has to pass a given sequence of network functions. Cohen et al. [7] considered NFV placement in an SDN with and without server capacity constraints for NFV-enabled unicast requests. They reduced their problems into incapacitated and capacitated facility location problems respectively, and provided a bi-criteria approximation solution to the problems. Kuo et al. [21] studied how to implement a single NFV-enabled unicast request with the end-to-end delay constraint by proposing a dynamic programming solution to the problem. Guo et al. [11] investigated the throughput maximization problem in NFV-enabled SDNs with respect to service chaining specifications. They also studied the online version that assumes the future arrivals of requests are not known in advance. Xu et al. [39] studied the throughput maximization and resource optimization problem in 
NFV-enabled networks, by assuming that the instances of different types of service chains have been instantiated in data centers. An optimal solution is proposed for a special case of the problem, and an approximation algorithm is devised for the original problem.

There are several studies focusing on multicasting in SDNs [15], [42], [43]. Huang et al. [16] recently devised the first online algorithms with provable competitive ratios for online unicasting and multicasting in SDNs, under both node capacities (forwarding table sizes) and link capacities (bandwidth capacities) constraints. However, they did not consider network function requirements of unicast and multicast requests. Huang et al. [15] studied the scalability of multicasting in SDNs, by proposing an efficient algorithm to find a branch-aware Steiner Tree (BST) for each multicast request. Their solution is not applicable to the NFV-enabled multicasting problem, due to the lack of efficient methods to deal with NFV placements. A very closely related work to this study is the one by Zhang et al. [42], [43]. They investigated the NFV-enabled multicasting problem in SDNs, by assuming that there are sufficient computing and bandwidth resources to accommodate any multicast request, for which they provided a 2-approximation algorithm for the problem if only one server $(M=1)$ is deployed for implementing the service chain of each multicast request. However, their method cannot be extended to a general case of the problem where multiple servers are employed. It must be mentioned that for the sake of reliability, it is usually better to have the traffic of each multicast being processed by multiple servers $(M>1)$. In case of the failure of a server, the other servers can continue the data traffic of the request without being interrupted. Furthermore, in reality, it is not uncommon that both computing and bandwidth resources in an SDN are limited, and they need to be carefully allocated. Furthermore, Zhang et al. [42], [43] did not consider dynamic admissions of a sequence of multicast requests, which is much complicated compared with admitting one or a set of given requests. Since the resources allocated to current requests will heavily impact the admissions of future requests and different requests may have different resource demands, it becomes very crucial that which requests should be admitted/rejected to maximize the network throughput.

Unlike most existing studies on online multicast routing problems in traditional networks that considered either the node capacity [17], [24], [25] or the link bandwidth constraint [2], [32], we take into account both the computing resource capacity of servers and the bandwidth constraint on links in an SDN. This is a much more challenging optimization problem, due to (1) the need of innovative cost models that can accurately capture the usage costs of two different types of resources and new techniques to analyze the performance of proposed online algorithms; and (2) the need of jointly considering placing virtualized network functions to the servers and finding multicast routes for each multicast request. In addition, our problem is much more general than the Steiner tree problem, because we need to not only the server locations of VNFs but also the routing graph consisting of the server locations. Therefore, traditional Steiner tree algorithms, such as Kou et al.'s algorithm [20], cannot be directly applicable for the NFV- enabled multicasting problem.

\section{Preliminaries}

In this section we first introduce the system model, notations and notions, and then define the problems.

\subsection{System model}

We consider a software-defined network $G=(V, E)$ with a set $V$ of SDN-enabled switch nodes and a set $E$ of links between SDN-enabled switch nodes. Some of the switch nodes in $V$ are attached with computing servers that can implement various network functions as virtual machines (VMs). The communication delay between a switch node and the server attached to it usually is negligible in comparison with the communication delay with other nodes in the network, as they are connected by a high-speed optical fiber. We thus denote by $V_{S}(\subseteq V)$ the subset of switch nodes attached with servers. Notice that each node $v \in V_{S}$ is treated as a switch node without an attached server if its server is not used for implementing VMs. Otherwise, the VM implementation cost of $v$ must be taken into account. Denote by $C_{v}$ and $B_{e}$ the computing capacity of the server attached to switch node $v \in V_{S}$ and the bandwidth capacity of link $e \in E$ in $G$, respectively. There is an SDN controller in $G$ that controls the allocations of both computing and bandwidth resources of $G$ to meet the resource demands of each admitted NFV-enabled multicast request. Specifically, when an SDN switch does not know how to handle the first packet of a newly arrived multicast request, it will send a message to the controller. Based on the resource availabilities of links and nodes that are collected by the controller, a route for the request will be calculated and installed in the switches in the route. Fig. 1 is an example of an SDN, where switch nodes $v_{1}, v_{2}$, and $v_{6}$ are attached with servers, while the rest of its nodes are not.

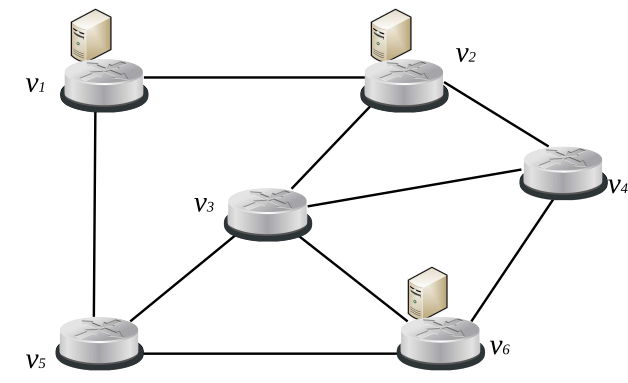

(a) An SDN $G$ with a set $V=$ $\left\{v_{1}, v_{2}, v_{3}, v_{4}, v_{5}, v_{6}\right\}$ of SDN-enabled switches and a subset $V_{S}=\left\{v_{1}, v_{2}, v_{6}\right\}$ of switches that are attached with servers.

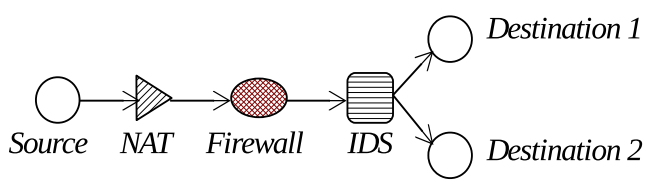

(b) A service chain $\langle$ NAT, Firewall, IDS $\rangle$.

Fig. 1. System model and a service chain example 


\subsection{NFV-enabled multicast requests and pseudo- multicast trees}

An NFV-enabled multicast request $r_{k}$ is represented by a quadruple $r_{k}=\left(s_{k}, D_{k} ; b_{k}, S C_{k}\right)$, where $s_{k} \in V$ is the source, $D_{k}$ is the set of destinations (or terminals) with $D_{k} \subseteq V, b_{k}$ is the demanded bandwidth by $r_{k}$, and $S C_{k}$ is the service chain of $r_{k}$ that consists of a sequence of network functions that must be implemented by either dedicated hardware middleboxes or virtual machines. Specifically, the service chain $S C_{k}$ of request $r_{k}$ enforces that every packet from the source of $r_{k}$ to go through each of the network functions of the service chain in the specified order prior to reaching its destinations, as illustrated in Fig. 1 (b). The network functions in $S C_{k}$ can be implemented by VMs in servers [12], [29], [33], [40]. Without loss of generality, we assume that the network functions in $S C_{k}$ are consolidated to a server in $G$. Specifically, when realizing multicast request $r_{k}$, its packet passes a server hosting the VMs of its service chain $S C_{k}$, the traffic will be directed to the VMs. Denote by $C_{v}\left(S C_{k}\right)$ the amount of demanded computing resource to implement $S C_{k}$ of multicast request $r_{k}$ in server $v \in V_{S}$.

A pseudo-multicast tree in $G$ is a graph $G_{T}$ derived from a multicast tree $T$ for the data traffic routing of an NFVenabled multicast request. We here use an example to illustrate the pseudo-multicast tree concept.

Consider a multicast tree $T$ as shown in Fig. 2, where nodes $A$ and $B$ are attached with servers for processing the NFVs in $S C_{k}$ of a multicast request $r_{k}$, the set of destinations is $\left\{d_{1}, d_{2}, d_{3}, d_{4}, d_{5}\right\}$. Recall that a packet from source $s_{k}$ must pass through a server for processing the NFVs in $S C_{k}$ prior to reaching all destinations. However, in this case, only the destinations $d_{1}$ and $d_{4}$ in $T$ can correctly receive the processed packet, because there are servers at $A$ and $B$ respectively, while the other three destinations $d_{2}, d_{3}$ and $d_{5}$ cannot. To enable the packet to pass through a server before reaching $d_{2}, d_{3}$ and $d_{4}$, the packet routing proceeds as follows. When the packet is processed in node $A$, the processed packet is sent back to node $a$ along the tree path $P_{A, a}$, node $a$ then forwards the processed packet to $d_{2}$ (see Fig. 2(b)). Similarly, the processed packet at $B$ will be sent back to node $b$ along the tree path $P_{B, b}$. Assume that the distance between nodes $A$ and $e$ is greater than the distance between nodes $B$ and $e$, the processed packet at node $b$ will be further forwarded to node $e$. The processed packet will finally reach node $e$ and be forwarded to node $d_{5}$ (see Fig. 2 (b)). We term this routing graph derived from $T$ as a pseudo-multicast tree $G_{T}$, as shown by Fig. 2 (b). It can be seen that another tree $T^{\prime}$ (see Fig. 2 (c)) derived from $G_{T}$ will have the same cost as $G_{T}$, i.e., $c\left(T^{\prime}\right)=c\left(G_{T}\right)$.

Notice that given an NFV-enabled multicast request, its pseudo-multicast tree may not be unique, because its packet can be directed to different destinations via different paths. However, the determination of a pseudo-multicast tree can be tailored to fit different optimization objectives. For example, if the objective is to minimize the cost of implementing the request, the found pseudo-multicast tree should be able to achieve the lowest cost.

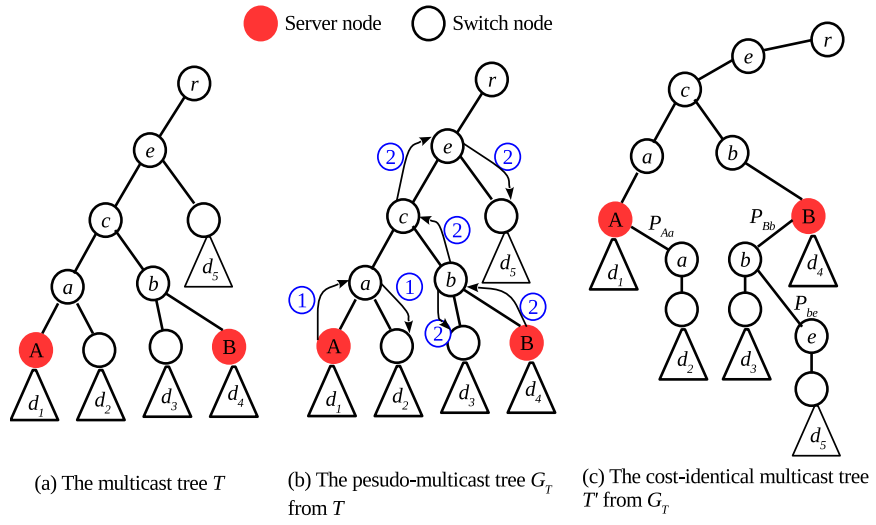

Fig. 2. A pseudo-multicast tree $G_{T}$ derived from a multicast tree $T$ for an NFV-Enabled multicast request $r_{k}$, and another tree $T^{\prime}$ derived from $G_{T}$ which has the identical cost as $G_{T}$.

\subsection{Problem definitions}

Given an $\operatorname{SDN} G=(V, E)$ and a multicast request $r_{k}$ $\left(=\left(s_{k}, D_{k} ; b_{k}, S C_{k}\right)\right)$, we consider three NFV-enabled multicasting problems with and without resource capacity constraints as follows.

Problem 1. Assuming that $G=(V, E)$ has sufficient computing and bandwidth resources to meet the resource demands of any NFV-enabled multicast request, the network operator of $G$ charges each admitted multicast request on a pay-as-you-go basis, its major concern is its operational cost that is defined as the sum of costs of consumed computing and bandwidth resources by all admitted requests. Let $c_{e}$ and $c_{v}$ be the usage costs of one unit of bandwidth and computing resources at link $e \in E$ and server $v \in V_{S}$, respectively. Denote by $T_{k}$ the pseudo-multicast tree for a multicast request $r_{k}$, the implementation cost of $r_{k}$ is defined by

$$
c\left(T_{k}\right)=b_{k} \cdot \sum_{e \in T_{k}} c_{e}+C_{v}\left(S C_{k}\right) \sum_{v \in V_{S}^{k}} c_{v},
$$

where $V_{S}^{k}$ is the set of servers that implement the service chain of request $r_{k}$. Notice that the edge cost $b_{k} \cdot \sum_{e \in T_{k}} c_{e}$ is incurred due to the consumption of bandwidth resource.

Notice that one edge in $G$ may be traversed more than once in the pseudo-multicast tree, and each time its usage cost is counted. Since the computing resource demand of the service chain of each request usually is no greater than the computing capacity of each server, we assume that the number of servers, each of which implements the VNFs of the service chain $S C_{k}$ of a single multicast request $r_{k}$, is no more than a constant $M$ with $M \geq 1$.

The NFV-enabled multicasting problem without SDN resource capacity constraints in $G$ for an NFV-enabled multicast request $r_{k}$ is to find a pseudo-multicast tree such that its implementation cost is minimized, if no more than $M$ servers are used for implementing its service chain $S C_{k}$, assuming that $G$ has sufficient computing and bandwidth resources for the request.

Problem 2. Both computing and communication resources in $G$ are capacitated. Then, for an incoming NFVenabled multicast request, the network may or may not have enough resources at that moment to admit the request. Or it is too expensive to admit the request, i.e., the request should be rejected. 
The NFV-enabled multicasting problem with SDN resource capacity constraint in $G$ for a single NFV-enabled multicast request $r_{k}$ is to find a pseudo-multicast tree for the request such that its implementation cost is minimized, if no more than $M$ servers are used for implementing its service chain $S C_{k}$, subject to the computing and bandwidth capacities in $G$.

Notice that the both defined problems are NP-hard, as their special case - the traditional multicast problem without service chain requirements is NP-hard [23].

Problem 3. In reality, requests arrive into the network one by one without the knowledge of future request arrivals. We refer to this dynamic request admission as online request admissions. As $G$ is a service SDN and open for public access, all of its computing and bandwidth resources are dynamically allocated to users based on the 'pay-as-you-go' principle, its resources may not always meet the resource demands of all requests at any time, while each arrived request must be responded by either admitting or rejecting it immediately. We thus formulate dynamic admissions of NFV-enabled multicast requests as the online NFV-enabled multicasting problem in $G$ with the aim to admit as many as NFV-enabled multicast requests without the knowledge of future request arrivals, while meeting both computing and bandwidth resource demands of each admitted request, subject to both computing and bandwidth capacities on servers and links in $G$, assuming that no more than $M$ servers are used to implement the service chain of each request.

\subsection{Approximation and competitive ratios}

Due to NP-hardness of the defined problems, we will propose approximation algorithms with approximation ratios and online algorithms with competitive ratios for them, where the approximation and competitive ratios are defined as follows, respectively.

The approximation ratio: Given a value $\gamma \geq 1$, a $\gamma$ approximation algorithm for a minimization problem $P_{1}$ is a polynomial time algorithm $\mathcal{A}$ that outputs a solution whose value is no more than $\gamma$ times the value of an optimal solution for any instance $I$ of $P_{1}$, where $\gamma$ is termed as the approximation ratio of algorithm $\mathcal{A}$.

Let $O P T$ and $S$ be an optimal solution of the offline problem and the solution delivered by an online algorithm $\mathcal{A}^{\prime}$ for a maximization problem $P_{2}$ respectively, where a sequence of requests arrives one by one without the knowledge of future request arrivals. The competitive ratio of the online algorithm $\mathcal{A}^{\prime}$ is $\xi$ if $\frac{S}{O P T} \geq \frac{1}{\xi}$ for any instance $I$ of the maximization problem $P_{2}$.

For the sake of convenience, symbols used in this paper are summarized in Table 1.

\section{Approximation Algorithms for the NFV- enabled Multicasting Problem}

In this section we deal with the NFV-enabled multicasting problem with and without SDN resource capacity constraints.

\subsection{Algorithm overview}

The basic idea of the proposed approximation algorithms is to find a pseudo-multicast tree rooted at the source and spanning all destinations, and each packet from the source to destinations passes through a server in the tree, such that the cost of the tree is minimized. To this end, the finest trade-off between the computing and communication costs needs to be explored. Specifically, if a server $v$ with a lower computing cost is included in the pseudo-multicast tree for multicast request $r_{k}$, the computing cost of implementing $r_{k}$ may be reduced. This however will increase the communication cost if the location of server $v$ is far from the destinations of $r_{k}$. On the other hand, if there are multiple servers located at different branches of the multicast tree, then the packet can pass through each of the servers to reach its destinations in $D_{k}$. This will lead to less bandwidth usages from the source to the destinations, which is achieved at the expense of high computing cost by employing multiple servers. We thus need to identify a set of servers with each implementing the service chain $S C_{k}$ of $r_{k}$ and find a pseudo-multicast tree including the identified server(s) on the path from the source $s_{k}$ to each destination $u \in D_{k}$. As $M$ is a constant, we aim to find a pseudo-multicast tree in $G$ that contains no more than $M$ servers and the path in the tree from $s_{k}$ to each destination $u \in D_{k}$ must pass through one of the identified servers such that the cost of the tree is minimized.

Recall that there are $\left|V_{S}\right|$ switches in $G$ with servers, clearly $M \leq\left|V_{S}\right|$. As a pseudo-multicast tree for any NFVenabled multicast request can contain at least one but no more than $M$ servers, there are at most $\left(\begin{array}{c}\left|V_{S}\right| \\ M\end{array}\right)$ combinations of servers that can meet the computing resource demand of service chain $S C_{k}$ of request $r_{k}$. For each combination of servers, a pseudo-multicast tree in $G$ can be identified, and the tree with the minimum cost is then used to implement $r_{k}$. We thus reduce the NFV-enabled multicast problem to a Steiner tree problem in an auxiliary undirected graph. An approximate solution to the latter returns an approximate solution to the former.

\subsection{Approximation algorithm without resource capacity constraints}

Given an NFV-enabled multicast request $r_{k}$, we now devise an approximation algorithm for the NFV-enabled multicasting problem in $G$ without SDN resource capacity constraint, by reducing it to the Steiner tree problem in an auxiliary undirected graph $G_{k}^{i}=\left(V_{k}^{i}, E_{k}^{i} ; c\right)$ with an edge weight function $c$ for all $i$ with $1 \leq i \leq\left(\begin{array}{c}\left|V_{S}\right| \\ M\end{array}\right)$, where $V_{k}^{i}=V \cup\left\{s_{k}^{\prime}\right\}$, $E_{k}^{i}=E \cup\left\{\left(s_{k}^{\prime}, v\right) \mid v \in V_{S}^{i}\right\}, V_{S}^{i}\left(\subseteq V_{S}\right)$ is the $i$ th combination of servers in $V_{S}$, and $s_{k}^{\prime}$ is a virtual source of request $r_{k}$. For each $v \in V_{S}^{i}$, if edge $\left(s_{k}, v\right) \in E$ in $G$, the cost of edge $\left(s_{k}, v\right) \in E_{k}^{i}$ is assigned zero. $s_{k}^{\prime}$ is the new source in $G_{k}^{i}$, replacing the original source $s_{k}$. Notice that the original source $s_{k}$ is still contained in $G_{k}^{i}$ serving as a 'regular' switch node without an attached server. To guarantee that the traffic of $r_{k}$ passes through its service chain $S C_{k}$ that is implemented in one or multiple servers in $V_{S}^{i}\left(\subseteq V_{S}\right)$, we connect $s_{k}^{\prime}$ with all server nodes in $V_{S}^{i}$, where the edge between $s_{k}^{\prime}$ and each server node $v \in V_{S}^{i}$ in $G_{k}^{i}$ represents a shortest path $p_{s_{k}, v}$ in $G$ between nodes 


\begin{tabular}{|c|c|}
\hline Symbols & Meaning \\
\hline$G=(V, E)$ & a software-defined network with a set $V$ of switches and a set $E$ of links \\
\hline$v$ and $e$ & a switch node $v \in V$ and a link $e \in E$ \\
\hline$V_{S}(\subset V)$ & the subset of switches nodes attached with servers \\
\hline$C_{v}$ and $B_{e}$ & computer capacity of the server attached to node $v \in V_{S}$ and the bandwidth capacity of link $e \in E$ \\
\hline$r_{k}=\left(s_{k}, D_{k} ; b_{k}, S C_{k}\right)$ & $\begin{array}{l}\text { a request with source node } s_{k} \text {, a set } D_{k} \text { of destination (or terminals), an amount } b_{k} \text { of bandwidth resource } \\
\text { demand, and service chain requirement } S C_{k}\end{array}$ \\
\hline$C_{v}\left(S C_{k}\right)$ & $\begin{array}{l}\text { the amount of demanded computing resource to implement } S C_{k} \text { of multicast request } r_{k} \text { in the server that is } \\
\text { attached to } v \in V_{S} \text {. }\end{array}$ \\
\hline$T$ and $G_{T}$ & a multicast tree and the pseudo-multicast tree derived from it \\
\hline$c(T)$ & the total edge of a multicast tree $T$ \\
\hline$c_{e}$ and $c_{v}$ & the usage costs of one unit of bandwidth and computing resources at link $e \in E$ and server $v \in V_{S}$ \\
\hline & the set of servers that implement the service chain of request $r_{k}$ \\
\hline & ticast tree for $r_{k}$ \\
\hline & the maximum number of servers that can be used to implement the service chain of each request \\
\hline & the approximation ratio of an approximation algorithm \\
\hline$O P T$ and $S$ & an optimal solution to the offline problem and the solution delivered by an online algorithm for a maximization \\
\hline$S / O P T$ & the competitive ratio of an online algorithm $\mathcal{A}^{\prime}$ \\
\hline$G_{k}^{i}=\left(V_{k}^{i}, E_{k}^{i} ; c\right)$ & $\begin{array}{l}\text { the } i \text { th auxiliary graph constructed from graph } G \text { for request } r_{k} \text {, where } 1 \leq i \leq\left(\begin{array}{c}\left|V_{S}\right| \\
M\end{array}\right), V_{k}^{i} \text { is node set, } E_{k}^{i} \text { is } \\
\text { edge set, and } c \text { is an edge weight function }\end{array}$ \\
\hline & a virtual source node of $r_{k}$ \\
\hline$T_{m s t}^{i}$ & the minimum spanning tree in a complete graph consisting of nodes in $\left\{s_{k}^{\prime}\right\} \cup D_{k}$ \\
\hline$H_{k}^{i}$ & the subgraph of $G_{k}^{i}$ derived from $T_{m s t}^{i}$ \\
\hline $\begin{array}{l}T_{k}^{i} \\
G^{\prime}\end{array}$ & $\begin{array}{l}\text { Steiner tree in } H_{k}^{i} \\
\text { a subgraph of } G \text { where edges and node with insufficient available resources are removed, which is used in }\end{array}$ \\
\hline$C_{v}(k)$ and $B_{e}(k)$ & $\begin{array}{l}\text { the amount of available computing and bandwidth resources at node } v \text { and link } e \text {, when multicast request } r_{k} \\
\text { arrives }\end{array}$ \\
\hline$c_{v}(k)$ and $c_{e}(k)$ & the unit resource usage costs at server node $v$ and link $e$ of the $k$ th request $r_{k}$ \\
\hline $\begin{array}{l}\alpha \text { and } \beta \\
V_{S}(k)\end{array}$ & $\begin{array}{l}\text { constant bases in exponential functions } \\
=\left\{v \mid v \in V_{S}, C_{v}(k)>C_{v}\left(S C_{k}\right)\right\}\end{array}$ \\
\hline$w(e)$ & weight of an edge in the auxiliary graph $G_{k}^{i}$ \\
\hline$v^{\prime}$ and $v^{\prime \prime}$ & virtual nodes for server $v$ \\
\hline$\sigma_{v}$ and $\sigma_{e}$ & two thresholds for node and edge costs of implementing a multicast request \\
\hline$P_{x, y}^{T}$ & a path in $T$ between node $x$ and node $y$ \\
\hline & lests \\
\hline
\end{tabular}

$s_{k}$ and $v$. The weight of edge $\left(s_{k}^{\prime}, v\right)$ is the cost sum of the edges in path $p_{s_{k}, v}$ plus the cost of implementing $S C_{k}$ in server $v$, i.e., $c_{\left(s_{k}^{\prime}, v\right)}=\sum_{e \in p_{s_{k}^{\prime}, v}} c_{e} \cdot b_{k}+c_{v}\left(S C_{k}\right)$, where $c_{v}\left(S C_{k}\right)$ is the cost of the amount $C_{v}\left(S C_{k}\right)$ of computing resource consumption for implementing $S C_{k}$. In addition, the weight $c_{e}$ of each edge $e \in E_{k}^{i} \cap E$ is the cost $c_{e} \cdot b_{k}$ of allocating the amount $b_{k}$ of bandwidth resource to request $r_{k}$ on edge $e \in E$. An example of the constructed auxiliary graph $G_{k}^{i}$ that is derived from the SDN in Fig. 1 is shown in Fig. 3.

For the sake of convenience, in the rest of this paper we assume that $V_{S}=\left\{v_{1}, v_{2}, \ldots, v_{\left|V_{S}\right|}\right\}$. Having constructed the auxiliary graph $G_{k}^{i}$, we now find a Steiner tree in $G_{k}^{i}$ for request $r_{k}$. We first find a minimum spanning tree (MST) $T_{m s t}^{i}$ in a complete graph consisting of nodes in $\left\{s_{k}^{\prime}\right\} \cup D_{k}$, in which each edge is assigned a weight that is equal to the length of the shortest path in $G_{k}^{i}$ between its two endpoints. Let $H_{k}^{i}$ be a subgraph of $G_{k}^{i}$ derived from $T_{m s t}^{i}$ by replacing each edge of $T_{m s t}^{i}$ with its corresponding shortest path in $G_{k}^{i}$. We then find an approximate Steiner tree $T_{k}^{i}$ in $H_{k}^{i}$, by applying the approximation algorithm due to Kou et al. [20], which will serve as the pseudo-multicast tree for $r_{k}$. Although there are more efficient approximation algorithms for the Steiner problem such as [4], [35], either they are randomized algorithms and their solutions are based on randomized rounding, which may not be feasible, or they

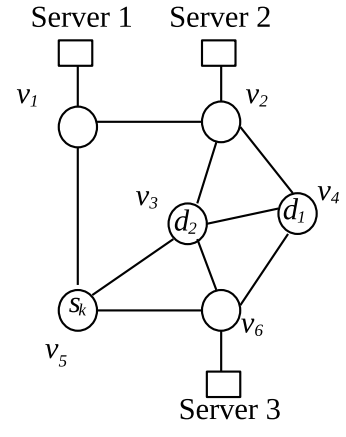

$G=(V, E)$

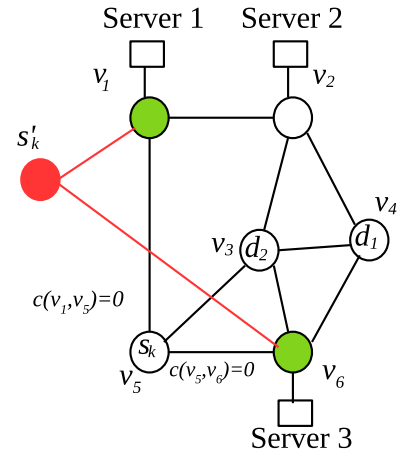

$G_{k}^{5}=\left(V_{k}^{5}, E_{k}^{5}\right)$
Fig. 3. An example of the auxiliary graph $G_{k}^{5}=\left(V_{k}^{5}, E_{k}^{5}\right)$ constructed from an SDN $G=(V, E)$ with $V_{S}^{5}=\left\{v_{1}, v_{6}\right\}$, assuming that $M=2$ and $V_{S}=\left\{v_{1}, v_{2}, v_{6}\right\}$. There are $\left(\begin{array}{c}\left|\vec{V}_{S}\right| \\ M\end{array}\right)=3 \cdot 2=6$ auxiliary graphs derived from $G$, and all different combinations of servers in $V_{S}$ are $V_{S}^{1}=\left\{v_{1}\right\}$, $V_{S}^{2}=\left\{v_{2}\right\}, V_{S}^{3}=\left\{v_{6}\right\}, V_{S}^{4}=\left\{v_{1}, v_{2}\right\}, V_{S}^{5}=\left\{v_{1}, v_{6}\right\}$, and $V_{S}^{6}=$ $\left\{v_{2}, v_{6}\right\}$.

have theoretical interest by giving better approximation ratios but take a much longer running time, which may not be practical under our application scenario where each request must be responded immediately, by either admitting or rejecting the request; otherwise, users may not be happy to the service and will escape from the services. While Kou et al.'s algorithm can produce a quality solution in a reasonable amount of time, and the solution is no greater than twice 
the optimal one. Furthermore, Kou et al.'s algorithm can be easily implemented distributively.

The detailed algorithm for the NFV-enabled multicast problem without SDN resource capacity constraints is given in Algorithm 1.

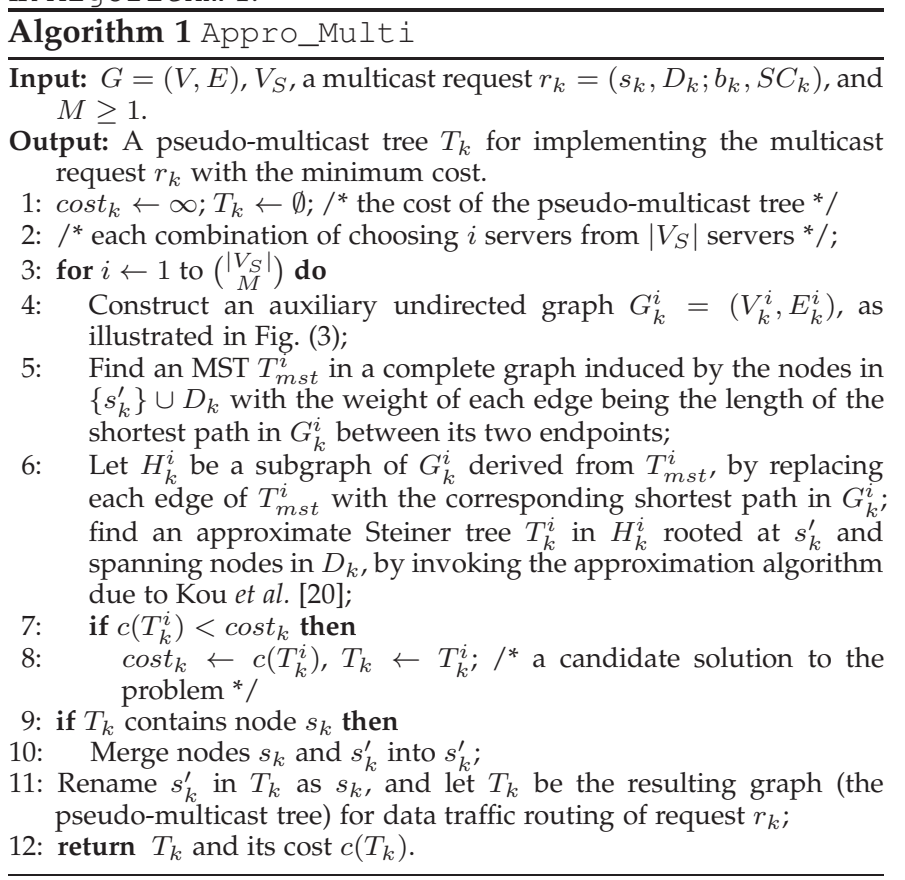

To illustrate the steps of Algorithm 1, we use $G_{k}^{5}$ in Fig. 3 as an example to show how the algorithm works in finding MST $T_{m s t}^{i}$ and $H_{k}^{i}$ in Fig. 4.

\subsection{Approximation algorithm with resource capacity constraints}

We now deal with the NFV-enabled multicasting problem under both computing and bandwidth resource capacity constraints, by performing minor modifications to Algorithm 1. Specifically, a subgraph $G^{\prime}=\left(V^{\prime}, E^{\prime}\right)$ of $G$ is constructed, where $V^{\prime}=V, E^{\prime}=\{(u, v) \mid(u, v) \in E$, and the residual bandwidth at link $(u, v)$ is no less than $\left.2 \cdot b_{k}\right\}$, a subset set $V_{S}^{\prime}$ of $V_{S}$ will be used, and $V_{S}^{\prime}=\left\{v_{i} \mid v_{i} \in V_{S}\right.$ if the available computing resource at $v_{i}$ can meet the computing resource demands of $\left.r_{k}\right\}$. Notice that link $e$ is in $E^{\prime}$ only if its residual bandwidth is no less than $2 b_{k}$. Specifically, an amount $b_{k}$ of bandwidth resource is reserved for the traffic of $r_{k}$ before being processed by its service chain. Another $b_{k}$ bandwidth resource is reserved to its traffic after being processed by its service chain in case the processed traffic traverse the same link of its original traffic. This is shown in Lemma 2.

Algorithm 1 then is applied to graph $G^{\prime}$, using the server set $V_{S}^{\prime}$. Clearly, all the resource demands by $r_{k}$ will be met. In case $G^{\prime}$ is disconnected, and none of its connected components contains the source and all destinations of $r_{k}$ and at least one server node, then the request should be rejected, because there are no sufficient resources in $G$ for its implementation. For simplicity, this algorithm is referred to as algorithm Appro_Multi_Cap.

Notice that in this paper we adopt a conservative way to perform resource allocation when admitting requests, by assuming that the system must have the sufficient resources to meet the resource demand of any admitted request $r_{k}$. However, reserving at least $2 b_{k}$ amounts of bandwidth in each link may exclude some links with less than $2 b_{k}$ but greater than $b_{k}$. Consequently, a few request may be rejected despite that there are sufficient resources for its admission. To this end, a greedy strategy can be adopted. That is, it first applies algorithm 1 in a subgraph with link residual capacity no less than $b_{k}$. If a pseudo-multicast tree for the request can be derived from the solution, then, the request is admitted. Otherwise, we apply algorithm 1 with each link of at least $2 b_{k}$ residual capacity for the request. If a pseudomulticast tree can be obtained, the request is admitted; it is rejected otherwise. In practice, a rejected request may not be rejected, and instead, the request can be placed to a waiting queue until it is admitted when the system has sufficient resources to meet its resource demands.

\subsection{Algorithm analysis}

The rest is to show the correctness of Algorithm Appro_Multi and Appro_Multi_Cap, and analyze its time complexity and the approximation ratio.

Lemma 1. Algorithm Appro_Multi delivers a feasible solution for the NFV-enabled multicasting problem with and without SDN resource capacity constraints.

See Appendix for the detailed proof.

To show the solution of algorithm Appro_Multi_Cap is feasible, we need to ensure that the solution delivered by it meets the network bandwidth capacity constraint on each link $e \in E$ as shown in Lemma 2.

Lemma 2. Given an SDN $G=(V, E)$ and a sequence of NFV-enabled multicast requests, let request $r_{k}=$ $\left(s_{k}, D_{k} ; b_{k}, S C_{k}\right)$ be the $k$ th multicast request, the solution delivered by algorithm Appro_Multi_Cap must preserve the network bandwidth capacity of each link, if the residual bandwidth of each edge in $G_{k}^{i}\left(V_{k}^{i}, E_{k}^{i} ; w\right)$ is no less than $2 b_{k}$ for all $i$ with $1 \leq i \leq\left(\begin{array}{c}\left|V_{S}\right| \\ M\end{array}\right)$.

See the Appendix for the detailed proof.

Theorem 1. Given an $\operatorname{SDN} G=(V, E)$, a set $V_{S}$ of switch nodes with each having an attached server, and an NFV-enabled multicast request $r_{k}=\left(s_{k}, D_{k} ; b_{k}, S C_{k}\right)$, there is a $2 M$ approximation algorithm, Algorithm 1, for the NFV-enabled multicasting problem with and without SDN resource capacity constraints, assuming no more than $M$ servers will be employed for its service chain implementation, where the approximation ratio $2 M$ is the best. The time complexity of the algorithm is $O\left(|V|^{3} \cdot\left|V_{S}\right|^{M}\right)$, where $\left|V_{S}\right| \ll|V|$ and $M \geq 1$ is a small integer.

See Appendix for the detailed proof.

\section{Online Algorithms for the OnLine NFV- enabled Multicasting Problem}

In this section we study the online NFV-enabled multicasting problem in $G$ with network resource capacity constraints. We first propose a novel cost model to capture dynamic resource consumptions in $G$. We then develop 


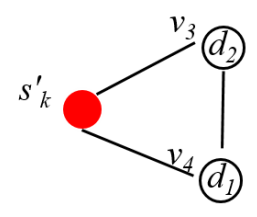

(a) A complete graph induced by the nodes in $\left\{s_{k}^{\prime}, d_{2}, d_{1}\right\}$.

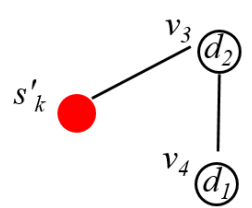

(b) A MST $T_{m s t}^{i}$ based on the complete graph built in (a)

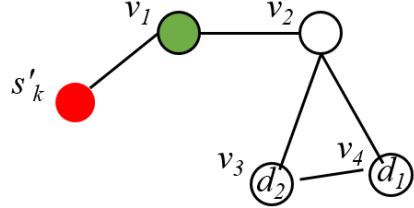

(c) A subgraph $H_{k}^{i}$ of $G_{k}^{5}$ constructed based on $T_{m s t}^{i}$ by replacing edges $\left(s_{k}^{\prime}, d_{2}\right)$ and $\left(d_{1}, d_{2}\right)$ in $T_{m s t}^{i}$ with paths $\left\{\left(s_{k}^{\prime}, v_{1}\right),\left(v_{1}, v_{2}\right),\left(v_{2}, v_{3}\right)\right\}$ and $\left\{\left(v_{4}, v_{2}\right),\left(v_{2}, v_{3}\right)\right\}$ in $G_{k}^{5}$, respectively.

Fig. 4. An example of steps of algorithm Appro_Multi based on auxiliary graph $G_{k}^{5}$ in Fig. 3 . Notice that edges $\left(s_{k}^{\prime}, d_{2}\right),\left(s_{k}^{\prime}, d_{1}\right)$, and $\left(d_{1}, d_{2}\right)$ in the complete graph of (a) corresponds to paths $p_{s_{k}^{\prime}, d_{2}}=\left\{\left(s_{k}^{\prime}, v_{1}\right),\left(v_{1}, v_{2}\right),\left(v_{2}, v_{3}\right)\right\}, p_{s_{k}^{\prime}, d_{1}}=\left\{\left(s_{k}^{\prime}, v_{6}\right),\left(v_{6}, v_{4}\right)\right\}$, and $p_{d_{1}, d_{2}}=\left\{\left(v_{4}, v_{2}\right),\left(v_{2}, v_{3}\right)\right\}$ in $G_{k}^{5}$, respectively

an efficient online heuristic for the problem based on the proposed cost model. We finally devise an online algorithm with a competitive ratio for a special case of the problem when $M=1$.

\subsection{Cost model}

Given an SDN $G=(V, E)$ with limited computing and bandwidth capacities at its servers and links, there is a need of a cost model to capture dynamic consumption of these resources in order to better guide the admissions of future requests and utilize the resources. A simple cost model, referred to the linear cost model, is widely adopted to assign each request with a cost that is proportional to the amount of its resource consumption regardless of whether a specific resource is overloaded or under-loaded. Clearly, this model may lead to some resources being underutilized while others being over-utilized. Consequently, the significant number of requests may be rejected due to unbalanced resource utilization. Intuitively, overloaded resources usually have higher probabilities of violating the resource demands of admitted requests, due to the high dynamics of resource consumptions. This eventually will affect the admissions of future requests. Therefore, we encourage the use of under-loaded resources while restricting the use of overloaded resources to maximize the number of NFVenabled multicast request admissions.

Motivated by the above concern, we here introduce a novel cost model that assigns an under-loaded resource with a lower cost and an overloaded resource with a higher cost. Thus, the resources in the network can be maximally allocated among user requests, thereby maximizing the network throughput. Specifically, let $C_{v}(k)$ be the amount of available computing resource at the server attached to a switch node $v \in V_{S}$ and $B_{e}(k)$ the amount of available bandwidth at link $e \in E$, respectively, when multicast request $r_{k}$ arrives.

To capture the resource use of $r_{k}$, we use exponential functions to represent the costs $c_{v}(k)$ and $c_{e}(k)$ of its usages of both computing and bandwidth resources at server node $v$ and link $e$ :

$$
c_{v}(k)=C_{v}\left(\alpha^{1-\frac{C_{v}(k)}{C_{v}}}-1\right),
$$

where $\alpha$ is a constant with $\alpha>1, C_{v}(k)=C_{v}(k-$ $1)-C_{v}\left(S C_{k}\right)$ if $r_{k}$ is admitted and $C_{v}(0)=C_{v}$ initially. $\left(1-\frac{C_{v}(k)}{C_{v}}\right)$ in Eq. (2) is the utilization ratio of computing resource at server $v \in V_{S}$. The rationale behind is that the use of less residual computing resource will be charged with a higher cost, while the use of plenty of residual computing resource will be charged with a much less cost.

The cost $c_{e}(k)$ of using the bandwidth resource at link $e$ prior to the admission of $r_{k}$ can be similarly defined, i.e.,

$$
c_{e}(k)=B_{e}\left(\beta^{1-\frac{B_{e}(k)}{B_{e}}}-1\right),
$$

where $\beta$ is a constant with $\beta>1, B_{e}(k)=B_{e}(k-1)-b_{k}$ if $r_{k}$ is admitted, and $B_{e}(0)=B_{e}$ initially. Notice that the values of $\alpha$ and $\beta$ reflect the sensitivity of resource usages. A larger value implies that its utilization of a specific resource is more sensitive.

\subsection{Online algorithm}

For each incoming multicast request $r_{k} \quad(=$ $\left.\left(s_{k}, D_{k} ; b_{k}, S C_{k}\right)\right)$, denote by $V_{S}(k)$ the subset of servers in $V_{S}$ that have sufficient residual computing resources to implement its service chain $S C_{k}$, i.e., $V_{S}(k)=\left\{v \mid v \in V_{S}, C_{v}(k) \geq C_{v}\left(S C_{k}\right)\right\}$. It thus follows that there are $\left(\begin{array}{c}\left|V_{S}(k)\right| \\ M\end{array}\right)$ combinations of servers that can meet the computing resource demand of the service chain $S C_{k}$. For each combination of servers in $V_{S}^{i}$, a pseudo-multicast tree $T_{k}^{i}$ in an undirected auxiliary graph $G_{k}^{i}=\left(V_{k}^{i}, E_{k}^{i} ; w\right)$ can be identified, and a pseudo-multicast tree with the minimum weight among all $T_{k}^{i}$ with $1 \leq i \leq\left(\begin{array}{c}\left|V_{S}(k)\right| \\ M\end{array}\right)$ is then used for the request implementation. The construction of $G_{k}^{i}=\left(V_{k}^{i}, E_{k}^{i} ; w\right)$ is given as follows.

Given an SDN $G=(V, E)$ and an NFV-enabled multicast request $r_{k}=\left(s_{k}, D_{k} ; b_{k}, S C_{k}\right)$, we construct an auxiliary undirected graph $G_{k}^{i}=\left(V_{k}^{i}, E_{k}^{i} ; w\right)$ for all $i$ with $1 \leq$ $i \leq\left(\begin{array}{c}\left|V_{S}(k)\right| \\ M\end{array}\right)$, where $V_{k}^{i}=V \cup\left\{s_{k}^{\prime}\right\} \cup\left\{v^{\prime}, v^{\prime \prime} \mid v \in V_{S}^{i}\right\}$ and $V_{S}(k) \subseteq$ ᄃ $\left.V\right) s=\left\{v \mid v \in V_{S}\right.$ and $\left.C_{v}(k) \geq c_{v}\left(S C_{k}\right)\right\}, E_{k}^{i}=$ $\left\{e \mid e \in E\right.$ and $\left.B_{e}(k) \geq 2 b_{k}\right\} \cup\left\{\left(s_{k}^{\prime}, v^{\prime}\right),\left(v^{\prime}, v^{\prime \prime}\right) \mid v \in V_{S}^{i}\right\}$ $\cup\left\{\left(v^{\prime \prime}, u\right) \mid(v, u) \in E, v \in V_{S}^{i}, u \in V\right.$, and $\left.B_{e=(u, u)} \geq 2 b_{k}\right\}$, $V_{S}^{i}\left(\subseteq V_{S}(k)\right)$ is the $i$-th combination of the servers in $V_{S}(k)$, and $s_{k}^{\prime}$ is a virtual source of request $r_{k}$. Fig. 5 illustrates the construction of $G_{k}^{i}$ when $i=5$ and $M=2$. 


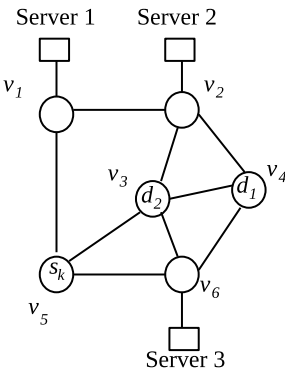

$G=(V, E)$

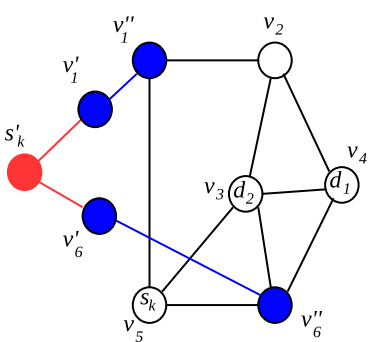

$G_{k}^{5}=\left(V_{R}^{5} E_{k}^{5}\right.$
Fig. 5. An example of the auxiliary graph $G_{k}^{5}=\left(V_{k}^{5}, E_{k}^{5} ; w\right)$ constructed from an SDN $G=(V, E)$ with $V_{S}^{5}(k)=\left\{v_{1}, v_{6}\right\}$ for an incoming NFVenabled multicast request $r_{k}$ that contains a source $s_{k}=v_{5}$ and a destination set $D_{k}=\left\{v_{3}, v_{4}\right\}$, assuming that $M=2$ and $V_{S}(k)=$ $\left\{v_{1}, v_{2}, v_{6}\right\}$. There are $\left(\begin{array}{c}\left|V_{S}\right| \\ M\end{array}\right)=3 \cdot 2=6$ auxiliary graphs derived from $G$ with each corresponding a different combination of servers in $V_{S}(k)$, i.e., $V_{S}^{1}=\left\{v_{1}\right\}, V_{S}^{2}=\left\{v_{2}\right\}, V_{S}^{3}=\left\{v_{6}\right\}, V_{S}^{4}=\left\{v_{1}, v_{2}\right\}, V_{S}^{5}=\left\{v_{1}, v_{6}\right\}$, and $V_{S}^{6}=\left\{v_{2}, v_{6}\right\}$.

Intuitively, each edge in $\left\{\left(s_{k}^{\prime}, v^{\prime}\right) \mid v \in V_{S}^{i}\right\}$ represents a shortest path $p_{s_{k}, v}$ in $G_{k}=\left(V_{k}, E_{k} ; w\right)$ between nodes $s_{k}$ and $v \in V_{S}$ while each edge in $\left\{\left(v^{\prime}, v^{\prime \prime}\right) \mid v \in V_{S}^{i}\right\}$ represents the server usage cost at $v$, where $V_{k}=V, E_{k}=E$, and $w(e)$ is the normalized cost of each edge $e \in E_{k}$ defined in Eq. (3). We then assign each edge $e \in E_{k}^{i}$ a weight in $G_{k}^{i}$ as follows.

$$
w(e)= \begin{cases}\sum_{e \in p_{s_{k}, v}} \frac{c_{e}(k)}{B_{e}} & \text { if } e=\left(s_{k}^{\prime}, v^{\prime}\right) \text { with } v \in V_{S}^{i}, \\ \frac{c_{v}(k)}{C_{v}}\left(c_{(v, u)}(k)\right. & \text { if } e=\left(v^{\prime}, v^{\prime \prime}\right) \text { with } v \in V_{S}^{i}, \\ B_{(u, v)} & \text { if } e=\left(v^{\prime \prime}, u\right) \text { with } v \in V_{S}^{i}, u \in V, \\ \frac{c_{e}(k)}{B_{e}} & \text { otherwise, } \quad(v, u) \in E,\end{cases}
$$

where $c_{v}(k)$ and $c_{e}(k)$ are the usage costs of computing resource at server node $v$ and the bandwidth resource at link $e \in E$ when request $r_{k}$ arrives, as defined in Eqs. (2) and (3), respectively. In other words, the differences between $G_{k}^{i}$ and $G$ are that we remove the edges without sufficient residual bandwidth to implement multicast request $r_{k}$, and add edges between virtual source $s_{k}^{\prime}$ and every $v^{\prime}$ (where $v \in V_{S}^{i}$ ) to represent a shortest path in $G$ between $s_{k}$ and $v$ and edges $\left(v^{\prime}, v^{\prime \prime}\right)$ to represent the resource usage cost of the server at $v$, and connect $v^{\prime \prime}$ to each neighbor node of $v$ in $G$. It is worth noting that since an amount $b_{k}$ of bandwidth resource along the edges in the shortest path $p_{s_{k}, v}$ for each $v \in V_{S}$ is reserved for the data traffic of $r_{k}$ from $s_{k}$ to server $v$ for processing, each edge $\left(v_{i}, v_{j}\right) \in p_{s_{k}, v}$ is in $E_{k}^{i}$ only if it has at least $b_{k}$ amounts of available bandwidth. For simplicity, we refer to the edges that are derived from switches in $V_{S}$ as node-derived edges. Denote by $E_{k, n o d e}^{i}$ the set of the nodederived edges. Clearly, $E_{k, \text { node }}^{i}=\left\{\left(v^{\prime}, v^{\prime \prime}\right) \mid \forall v \in V_{S}^{i}\right\}$.

Having constructed the auxiliary graph $G_{k}^{i}=$ $\left(V_{k}^{i}, E_{k}^{i} ; w\right)$, we find an approximate Steiner tree $T_{k}^{i}$ in $G_{k}^{i}$ by applying the approximation algorithm due to Kou et al. [20]. We use the same process to find an approximate Steiner tree $T_{k}^{i}$ for each value of $i$ with $1 \leq i \leq\left(\begin{array}{c}\mid V_{S}(k) \\ M\end{array}\right)$, i.e., different combinations of servers. Denote by $T_{k}^{\min }$ a pseudo-multicast tree with the minimum weight, i.e., $\sum_{e \in T_{k}^{\min }} w(e) \leq \sum_{e \in T_{k}^{i}} w(e)$ for all $i$ with $1 \leq i \leq\left(\begin{array}{c}V_{S}(k) \\ M\end{array}\right)$.

To decide whether request $r_{k}$ should be admitted, we here adopt a novel admission control policy that jointly considers both edge and node resource consumption costs, as the admission of $r_{k}$ will significantly impact the admissions of future requests whose arrivals are not known in advance. The rationale behind adopting an admission control policy is to control the impact of admissions of requests with large resource demands on the admissions of future requests. Specifically, if a request with a very large resource demand is admitted, the future requests may be rejected when it occupies the resource for a long time. This unfortunately will heavily reduce the network throughput. The admission control policy thus described as follows.

A multicast request $r_{k}$ will be admitted if it meets

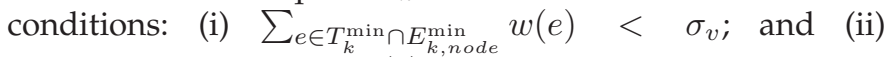
$\sum_{e \in T_{k}^{\min } \cap\left(E_{k}^{\min } \backslash E_{k, n \text { ode }}^{\min }\right)} w(e) \stackrel{k, \text { node }}{<} \sigma_{e}$, where $\sigma_{e}$ and $\sigma_{v}$ are the pre-defined admission control thresholds for both bandwidth and computing usage costs of $T_{k}^{\min }$, respectively.

If $T_{k}^{\mathrm{min}}$ does exist, a feasible solution to the original problem will be derived from $T_{k}^{\min }$, by replacing its each edge $\left(s_{k}^{\prime}, v^{\prime}\right)$ with the corresponding shortest path $p_{s_{k}, v}$ in $G$ and implementing the service chain $S C_{k}$ on the servers in $T_{k}^{\min }$. Note that no actual resource allocation is performed when finding $T_{k}^{i}$, and the resources are allocated to implement request $r_{k}$ only if $T_{k}^{i}$ meets the admission control conditions; otherwise, request $r_{k}$ will be rejected.

The detailed description of the algorithm is given in Algorithm 2.

\begin{tabular}{l} 
Algorithm 2 Online_Heu \\
\hline Input: An SDN $G=(V, E)$, a set $V_{S}$ of switches with each having \\
server attached to it, the bandwidth resource capacity $B_{e}$ of each \\
link $e \in E$ and computing resource capacity $C_{v}$ of each $v \in V_{S}$, \\
a sequence of NFV-enabled multicast requests $r_{k}$ that arrive at \\
the network one by one, and values for the pre-defined admission \\
control thresholds $\sigma_{e}$ and $\sigma_{v}$. \\
Output: Admit or reject each incoming multicast request $r_{k}$. If admit-
\end{tabular} Output: Admit or reject each incoming multicast request $r_{k}$. If
ted, a pseudo-multicast tree for the request will be returned.

1: $\mathcal{G} \leftarrow \emptyset ; /{ }^{*}$ a set of pseudo-multicast trees */

2: for each multicast request $r_{k}$ do

3: $\operatorname{cost}_{k} \leftarrow \infty ; T_{k}^{\min } \leftarrow \emptyset ; /^{*}$ the cost of the pseudo-multicast tree * /

4: $\quad$ Find the shortest paths in $G_{k}=\left(V_{k}, E_{k} ; w\right)$ from $s_{k}$ to all nodes in $V_{S}$, and let $p_{s_{k}, v}$ be the found path from $s_{k}$ to a node $v \in V_{S}$; for $i \leftarrow 1$ to $\left(\begin{array}{c}\left|V_{S}\right| \\ M\end{array}\right)$ do

6: $\quad$ Construct an auxiliary undirected graph $G_{k}^{i}=\left(V_{k}^{i}, E_{k}^{i} ; w\right)$ as shown in Fig. 5;

7: $\quad$ Find an approximate Steiner tree $T_{k}^{i}$ in $G_{k}^{i}$ rooted at $s_{k}^{\prime}$ and spanning the nodes in $D_{k}$, by invoking the approximation algorithm due to Kou et al. [20];

if $c\left(T_{k}^{i}\right)<\operatorname{cost}_{k}$ then

cost $_{k} \leftarrow c\left(T_{k}^{i}\right)$;

$T_{k}^{\min } \leftarrow T_{k}^{i} ;{ }^{*}$ a candidate solution */

if $T_{k}^{\min }$ does not exist then

Rejec
else Reject multicast request $r_{k}$;

if $\left(\sum_{e \in T_{k}^{\min } \cap E_{k, n o d e}^{\min } w(e) \quad} w \quad \sigma_{v}\right)$ and $\left(\sum_{e \in T_{k}^{\min } \cap\left(E_{k}^{\min } \backslash E_{k, \text { node }}^{\min }\right)} w(e)<\sigma_{e}\right)$ then

An undirected graph $G_{k}^{\prime \prime}$ is derived from $T_{k}^{\text {min }}$ by replacing each edge $\left(s_{k}^{\prime}, v^{\prime}\right)$ in $T_{k}^{\text {min }}$ with its corresponding shortest path $p_{s_{k}, v}$ in $G$;

$\mathcal{G} \leftarrow \mathcal{G} \cup\left\{G_{k}^{\prime \prime}\right\}$

else

Reject multicast request $r_{k}$;

19: return $\mathcal{G}$.

Notice that to ensure that the solution delivered by Algorithm 2 meets the network bandwidth capacity constraint on each link $e \in E$, link $e$ is contained in $E_{k}^{i}$ only 
if its residual bandwidth is no less than $2 b_{k}$, as shown in Lemma 2.

We finally analyze the performance of Algorithm 2 by stating it in Theorem 2.

Theorem 2. Given an $\operatorname{SDN} G=(V, E)$ with the computing capacity $C_{v}$ for the server attached to each switch $v \in V_{S}$ and the bandwidth capacity $B_{e}$ for each link $e \in E$, and a sequence of multicast requests with the $k$ th multicast request being represented by $r_{k}=\left(s_{k}, D_{k} ; b_{k}, S C_{k}\right)$, Algorithm 2 deliver a feasible solution for the online NFV-enabled multicasting problem if the request sequence consists of $k$ NFV-enabled multicast requests, and takes $O\left(k|V|^{3}\left|V_{S}\right|^{M}\right)$ time, where $M$ is an integer constant which is the number of servers for the request service chain processing.

See the Appendix for the detailed proof.

\subsection{Online algorithm with $M=1$}

We now propose an online algorithm with a provable competitive ratio for a special online NFV-enabled multicasting problem where only a single server will be used to implement the service chain of each multicast request, i.e., $M=1$. The basic idea behind the algorithm is to determine whether every incoming NFV-enabled multicast request $r_{k}$ will be admitted or rejected, depending on a given admission control policy. If $r_{k}$ is admissible, the algorithm requires to jointly find a server with sufficient computing resource to implement the service chain $S C_{k}$ and a pseudo-multicast tree for $r_{k}$, such that the cost of implementing the request is minimized, subject to the resource capacity constraints on the network.

To find a pseudo-multicast tree for request $r_{k}$, we have an important observation: one of the $\left|V_{S}\right|$ servers must be contained in any pseudo-multicast tree for each request $r_{k}$, the pseudo-multicast tree must include the server as one of the destination nodes of $r_{k}$. Thus, we can find a Steiner tree in $G_{k}=\left(V_{k}, E_{k} ; w\right)$ for request $r_{k}$ with source $s_{k}$ and the destination set $D_{k} \cup\{v\}$, where $v \in V_{S}$ has sufficient computing resource. Notice that $G_{k}\left(V_{k}, E_{k} ; w\right)$ is an undirected graph that is identical to $G(V, E)$, i.e., $V_{k}=V$ and $E_{k}=E$. The weight $w_{e}(k)$ of each edge $e \in E_{k}$ is assigned a normalized cost of the cost defined by Eq. (3), that is, $w_{e}(k)=c_{e}(k) / B_{e}$, while the weight $w_{v}(k)$ of each node $v \in V_{S}$ for $r_{k}$ is the normalized cost of the cost defined by Eq. (2), i.e, $w_{e}(k)=c_{v}(k) / C_{v}$.

Let $T$ be an approximate Steiner tree in $G_{k}$ rooted at $s_{k}$ and spanning the terminals in $D_{k}$ by the approximation algorithm due to Kou et al. [20], where $G_{k}$ is the graph $G(V, E)$ that considered the first $k-1$ requests already, partial resources at its servers and links are occupied by some of the first $(k-1)$ requests at this moment. We build a pseudo-multicast tree for $r_{k}$. If server $v$ is in any path in $T$ from $s_{k}$ to each destination, $T$ is the pseudo-multicast tree, and its cost is no more than twice the optimal one [20]; otherwise, assume that the path between $s_{k}$ and a destination node $d$ does not contain any server $v$. Let $u$ be the Lowest Common Ancestor (LCA) in $T$ between nodes $v_{1}$ and $v_{2}$, i.e., $u=L C A\left(v_{1}, v_{2}\right)$. Then, when the packet from $s_{k}$ is sent to server $v$ for processing, the processed packet continues forwarding to all destinations in the subtree rooted at $v$; for the destination $d \in D_{k}$, the processed packet at node $v$ is then sent back to node $u$, which then forwards toward destination $d$. Clearly, in the worst scenario, the processed packet will be sent back to the source $s_{k}$ for multicasting.

Let $T$ and $T^{*}$ be the found approximate Steiner tree by the approximation algorithm in [20] and the optimal one. Denote by $T_{k}$ and $T_{k}^{*}$ the pseudo-multicast tree based on $T$ and the optimal multicast tree, respectively. The sum of the weights of edges in the pseudo-multicast tree $T_{k}$ based on $T$ and server $v$ is

$$
\begin{aligned}
& w\left(T_{k}\right)+w_{v}(k)=w(T)+w\left(P_{v, u}^{T}\right)+w_{v}(k) \leq w(T) \\
& +w\left(P_{v, s_{k}}^{T}\right)+w_{v}(k) \leq 2 w(T)+w_{v}(k) \leq 2\left(w(T)+w_{v}(k)\right) \\
& \leq 4 w\left(T^{*}\right)+2 w_{v}(k) \leq 4\left(w\left(T^{*}\right)+w_{v}(k)\right) \leq 4 O P T_{v},
\end{aligned}
$$

where $P_{x, y}^{T}$ is a path in $T$ between node $x$ and node $y$, and $O P T_{v}$ is the optimal cost of the pseudo-multicast tree using server $v$ as its service chain processing server. Thus, the optimal solution $O P T$ for request $r_{k}$ in $G_{k}$ thus is $O P T=\min _{v \in V_{S}}\left\{O P T_{v}\right\}$.

We then adopt the following admission control policy to guide the admission of each multicast request $r_{k}$ : (a) If $w_{v}(k) \geq \sigma_{v}$ for any $v \in V_{S} \cap T_{k}, r_{k}$ will be rejected; and (b) if $\sum_{e \in T_{k}} w_{e}(k) \geq \sigma_{e}, r_{k}$ will be rejected, where $T_{k}$ is a pseudo-multicast tree delivered by an algorithm in $G_{k}$ for $r_{k}, \sigma_{v}>0$ and $\sigma_{e}>0$ are admission control thresholds of computing and bandwidth respectively, and $\sigma_{v}=\sigma_{e}=|V|-1$. The detailed online algorithm, referred to as Online_CP, is given in Algorithm 3.

\section{Algorithm 3 Online_CP}

Input: $G=(V, E), V_{S}, B_{e}$ for each $e \in E, C_{v}$ for each $v \in V_{S}, \mathrm{a}$ sequence of multicast requests that arrive at the network one by one with each request $r_{k}=\left(s_{k}, D_{k} ; b_{k}, S C_{k}\right), \sigma_{e}$, and $\sigma_{v}$.

Output: The admission or rejection of each incoming NFV-enabled multicast request, if admitted, a pseudo-multicast tree for the request will be delivered.

1: $\mathcal{G} \leftarrow \emptyset ; /^{*}$ the pseudo-multicast trees for the admitted requests */

2: for each incoming request $r_{k}$ do

3: $\quad T_{k} \leftarrow \emptyset_{;} /{ }^{*}$ the pseudo-multicast tree for $r_{k}$ if it is existent $* /$

4: $\quad$ cost $\leftarrow \infty ; /^{*}$ the cost of the pseudo-multicast tree $T_{k}$ for $r_{k} *$ /

5: $\quad$ Construct an undirected graph $G_{k}=\left(V_{k}, E_{k} ; w\right)$, by assigning a normalized weight $w_{v}(k)$ for each each node $v \in V_{S}$ and a normalized weight $w_{e}(k)$ for each link $e \in E$;

6: $\quad$ for each $v \in V_{S}$ do

7: $\quad$ if $w_{v}(k)<\sigma_{v}$ then

8: $\quad$ Find an approximate Steiner tree $T$ in $G_{k}$ with the terminal set $\left\{s_{k}, v\right\} \cup D_{k}$ by the algorithm due to Kou et al. [20]; if $\sum_{e \in T} w_{e}(k)<\sigma_{e}$ then

Compute the lowest common ancestor $u \quad=\quad L C A\left(v, d_{1}, d_{2}, \ldots, d_{\left|D_{k}\right|}\right) \quad$ in $T$, where $\operatorname{LCA}\left(x_{1}, x_{2}, \ldots, x_{n}\right)=$ $L C A\left(L C A\left(x_{1}, x_{2}, \ldots, x_{n-1}\right), x_{n}\right)$;

11: Calculate the cost $\operatorname{cost}(k)$ of pseudo-multicast tree derived from $T$ for $r_{k}$;

$\begin{array}{ll}\text { 12: } & \operatorname{cost}(k) \leftarrow c(T)+c_{v}\left(S C_{k}\right)+c\left(p_{v, u}\right) ; \\ \text { 13: } & \text { if } \operatorname{cost}(k)<\operatorname{cost} \text { then }\end{array}$

$\begin{array}{ll}\text { 12: } & \operatorname{cost}(k) \leftarrow c(T)+c_{v}\left(S C_{k}\right)+c\left(p_{v, u}\right) ; \\ \text { 13: } & \text { if } \operatorname{cost}(k)<\operatorname{cost} \text { then }\end{array}$

14: $\quad T_{k} \leftarrow T, \operatorname{cost} \leftarrow \operatorname{cost}(k)$;

15: $\quad$ if $T_{k} \neq \emptyset$, then Admit request $r_{k}, \mathcal{G} \leftarrow \mathcal{G} \cup\left\{<r_{k}, T_{k}\right.$, cost $\left.>\right\}$; 16: else Reject request $r_{k}$;

17: return $\mathcal{G}$.

We finally analyze the competitive ratio of Algorithm 3. Let $\mathcal{S}(k)$ and $O P T$ be the sets of admitted multicast requests by Algorithm 3 and an optimal offline algorithm when multicast request $r_{k}$ arrives. Let $\mathcal{R}(k)$ be the set of multicast requests that are rejected by Algorithm 3 while admitted by the optimal offline algorithm. Then the competitive ratio of Algorithm 3 is $\frac{|\mathcal{S}(k)|}{|\mathcal{S}(k) \cap O P T|+|\mathcal{R}(k)|} \geq \frac{|\mathcal{S}(k)|}{|\mathcal{R}(k)|+|\mathcal{S}(k)|}$, since 
$O P T=\mathcal{R}(k) \cup(O P T \cap \mathcal{S}(k)) \subseteq \mathcal{R}(k) \cup S(k)$. Specifically, the analysis of the competitive ratio of Algorithm 3 proceeds as follows. We first show the upper bound on the accumulative computing and bandwidth resources occupied by admitted requests in $\mathcal{S}(k)$ in Lemma 3. We then prove the lower bound on the accumulative computing and bandwidth resources occupied by rejected requests in $\mathcal{R}(k)$ in Lemma 4 . We finally derive the competitive ratio by combining the results of the upper and lower bounds on admitted and rejected requests by the proposed on line algorithm in Theorem 3.

Lemma 3. When a multicast request $r_{k}$ arrives, the cost sum of all servers in $V_{S}$ is $\sum_{v \in V_{S}} c_{v}(k) \leq 2 \mathbb{C}(k) \cdot \log \alpha \cdot(|V|-$ $1)$ and the cost sum of all links in $E$ is $\sum_{e \in E} c_{e}(k) \leq$ $2 \mathbb{B}(k) \cdot \log \beta \cdot(|V|-1)$, respectively, provided that $b_{k^{\prime}} \leq$ $\frac{\min _{e \in E} B_{e}}{\log \beta}$ and $C_{v}\left(S C_{k^{\prime}}\right) \leq \frac{\min _{v \in V_{S}} C_{v}}{\log \alpha}$ with $1 \leq k^{\prime} \leq \bar{k}$, where $\mathbb{B}(k)$ and $\mathbb{C}(k)$ are the accumulative amounts of bandwidth and computing resources being occupied by the admitted requests in $\mathcal{S}(k)$, respectively.

See the Appendix for the proof.

In the following, we show the lower bound on the cost of a multicast request in $\mathcal{R}(k)$ that is rejected by Algorithm 3 but admitted by an optimal offline algorithm.

Lemma 4. For each NFV-enabled multicast request $r_{k^{\prime}} \in$ $\mathcal{R}(k)$, if $\alpha=\beta=2|V|$, we have

$$
w\left(T_{k^{\prime}}^{\prime}\right)+w_{v^{\prime}}\left(k^{\prime}\right) \geq \frac{|V|-1}{4},
$$

where $T_{k^{\prime}}^{\prime}$ is the Steiner tree found by the optimal offline algorithm to route the traffic of $r_{k^{\prime}}$, and $v^{\prime}$ is the switch in $T_{k^{\prime}}^{\prime}$ whose server is selected to implement server chain $S C_{k^{\prime}}$, assuming that both the following inequalities are met: $b_{k^{\prime}} \leq \frac{\min _{e \in E} B_{e}}{\log \beta}$, and $C_{v}\left(S C_{k^{\prime}}\right) \leq$ $\frac{\min _{v \in V_{S}} C_{v}}{\log \alpha}$ with $1 \leq k^{\prime} \leq k$.

See the proof in Appendix.

We finally analyze the competitive ratio of Algorithm 3.

Theorem 3. Given an SDN $G=(V, E)$ with computing capacity $C_{v}$ of each server node $v \in V_{S}$ and bandwidth capacity $B_{e}$ for each link $e \in E$, a sequence of NFVenabled multicast requests with the $k$ th multicast request $r_{k}$ being represented by a quadruple $\left(s_{k}, D_{k} ; b_{k}, S C_{k}\right)$, there is an online algorithm, Algorithm 3, with a competitive ratio of $O(\log |V|)$ for the online NFV-enabled multicasting problem if only one server is contained in the pseudo-multicast tree for the service chain implementation of the request, provided that $b_{k^{\prime}} \leq \frac{\min _{e \in E} B_{e}}{\log \beta}$ and $C_{v}\left(S C_{k^{\prime}}\right) \leq \frac{\min _{v \in V_{S}} C_{v}}{\log \alpha}$ with $1 \leq k^{\prime} \leq k$. The algorithm takes $O\left(k|V|^{3}\right)$ time if the request sequence contains $k$ NFV-enabled multicast requests.

See the proof in Appendix.

\section{Performance Evaluation}

In this section we evaluate the performance of the proposed algorithms through experimental simulation, using both synthetic networks generated by the GT-ITM tool and real networks, in a platform programmed using Java. We also investigate the impact of important parameters on the performance of the proposed algorithms.

\subsection{Environment settings}

We consider SDNs consisting of from 50 to 250 nodes, where each network is generated using GT-ITM [10]. The number of servers in each network is set to $10 \%$ of the network size, and they are randomly co-located with switches in the network. We also use real network topologies, i.e., GÉANT [8] and an ISP network from [38]. There are nine servers for the GÉANT topology as set in [12] and the number of servers in the ISP networks is provided by [33]. The bandwidth capacity of each link varies from 1,000 Mbps to $10,000 \mathrm{Mbps}$ [19], and the computing capacity of each server varies from 4,000 to $12,000 \mathrm{MHz}$ [13]. Five types of network functions, i.e., Firewall, Proxy, NAT, IDS, and Load Balancing, are considered, and their computing demands are adopted from [12], [29]. The source and destination nodes of each multicast request is randomly generated, the ratio of the maximum number $D_{\max }$ of destinations of a multicast request to the network size $|V|$ is randomly drawn in the range of $[0.05,0.2]$, and its bandwidth resource demand is randomly drawn in the range of $[50,200] M b p s$. Using the hourly rate (price) of a general purpose m3.xlarge Amazon EC2 instance as a reference, the computing resource usage cost is set at $\$ 0.25$ per $\mathrm{MHz}$. We assume that the cost of consuming a unit bandwidth resource is proportional to the length of a link, thus the cost of bandwidth resource usage of a network link varies between $\$ 0.002$ and $\$ 0.005$ per Mbps. We set both $\sigma_{e}$ and $\sigma_{v}$ at $|V|-1$. The maximum number $M$ of servers that can be used to implement the service chain of each multicast request is 3 . The running time of each algorithm is obtained based on a machine with a $3.40 \mathrm{GHz}$ Intel i7 Quad-core CPU and 16 GiB RAM. Unless otherwise specified, these parameters will be adopted in the default setting.

We evaluate the performance of algorithm Appro_Multi against the state-of-the-art - an algorithm in [42], referred to as algorithm Alg_one_Server, which only uses a single server to implement service chain $S C_{k}$ of each multicast request $r_{k}$. Namely, it first routes the traffic of $r_{k}$ to a server, and then finds a Minimum Spanning Tree (MST) of a complete graph $G_{c}$ containing the destinations of $r_{k}$, where the edge between two destinations in $G_{c}$ represents the shortest path between the two nodes in the original network. It then expands the MST into its corresponding subgraph in the original network. It finally selects the combination of server and subgraph with the minimum cost.

For the online algorithms, we study the performance of algorithms Online_Heu and Online_CP against those of a baseline heuristic SP. For multicast request $r_{k}$, algorithm SP first removes links and nodes that do not have enough available resources to admit $r_{k}$, and then assigns each link and each switch node in $V_{S}$ with the same weight. For each candidate server in $V_{S}$, it then finds a shortest path from $s_{k}$ to $v$ and a single-source shortest path tree rooted at $v$ and spanning all destinations of $r_{k}$. It finally uses a pseudo-multicast tree with the minimum cost for $r_{k}$. We also compare the performance of algorithms Online_Heu and Online_CP against their counterparts without the admission control policy, which are denoted by algorithms Online_Heu_NoAd and Online_CP_NoAd, 
respectively. Specifically, in algorithms Online_Heu_NoAd and Online_CP_NoAd the only condition that can reject a multicast request is the lack of available resources to fulfil the resource demands of the request. In the following experiments, we use the operational cost to represent the total cost of all admitted requests.

\subsection{Performance evaluation of approximation algo- rithms}

We first evaluate the performance of algorithm Appro_Multi against that of algorithm Alg_One_Server by varying the network size from 50 to 250 and the ratio of the maximum number $D_{\max }$ of destinations of each request to the network size $|V|$ from 0.05 to 0.2 . The operational cost and running time curves delivered by algorithms Appro_Multi and Alg_one_Server are drawn in Fig. 6, where the operational costs and running times are the average of admitting 1,000 NFV-enabled multicast requests. Specifically, we can see from Fig. 6 (a) that the operational cost by algorithm Appro_Multi is around $80 \%$ of that of algorithm Alg_one_Server. The reason is that algorithm Appro_Multi may use multiple servers that are close to the destinations of the request to implement the service chain of the request, which can significantly reduce the cost of bandwidth resource usage. Furthermore, it can be seen from the figure that the performance gap between the two algorithms becomes larger and larger, with the increase on the network size. The rationale behind is that algorithm Appro_Multi has more chances to select a set of servers that are closer to the destinations of each request, considering that more servers in larger networks are to be chosen. The similar performance behavior can be observed from Figs. 6(b) and (c). Furthermore, it can be seen from Figures 6 (d) - (f) that approximation algorithm Appro_Multi takes a slightly more time than that of algorithm Alg_one_Server, as different combinations of servers in $V_{S}$ are to be considered.

We then investigate the performance of approximation algorithms Appro_Multi and Alg_one_Server in real networks GÉANT and AS1755, by varying $\frac{D_{\max }}{|V|}$ from 0.05 to 0.2 . Fig. 7 shows that the operational costs and running times of both algorithms. It can be seen that the operational cost delivered by algorithm Appro_Multi is much lower than that by algorithm Alg_one_Server while taking a slightly more running time. For example, the operational cost by algorithm Appro_Multi in network AS1755 is around $30 \%$ lower than that of algorithm Alg_One_Server when $\frac{D_{\max }}{|V|}=0.15$ as shown in Fig. 7 (b).

The rest is to evaluate the performance of approximation algorithm Appro_Multi_Cap, by setting $\frac{D_{\max }}{|V|}$ at 0.2 . Notice that algorithm Alg_one_Server is not considered as a benchmark as it does not consider SDN resource capacity constraints. From Figure 8(a), we can see that the operational cost of algorithm Appro_Multi_Cap is larger than that of algorithm Appro_Multi. The reason is that the algorithm Appro_Multi_Cap excludes the servers and links without enough available resources from the consideration for an incoming multicast request, this may reduce the number of combinations of servers that can be explored to implement request.

\subsection{Performance evaluation of online algorithms}

We then evaluate the proposed online algorithms Online_Heu and Online_CP against algorithms SP, Online_Heu_NoAd, and Online_CP_NoAd, by varying the network size from 50 to 250 for a monitoring period that consists of 300 multicast requests. The numbers of admitted requests by algorithms Online_Heu and Online_CP are shown in Fig. 9. Specifically, we can see from Fig. 9 (a) that online algorithm Online_Heu admits at least twice numbers of requests admitted by algorithm SP. Although the total resource capacity of each network keeps increasing with the increase on its size, the number of requests admitted by each mentioned algorithm on it does not monotonically increase. This is because it is very likely that the locations of the destinations of each multicast request in the network are far away from each other, thereby increasing the rejection likelihood of the request, due to more bandwidth resource consumptions. In addition, we can see that algorithm Online_Heu admits more requests than that by algorithm Online_Heu_NoAd without the admission control policy. The rationale behind is that the admission control policy rejects requests with high resource demands to preserve the resourced for future requests with low resource demands. Similar performance results delivered by online algorithm Online_CP can be observed in Fig. 9 (b).

We now evaluate the performance of online algorithms Online_Heu and Online_CP against online algorithms SP, Online_Heu_NoAd, and Online_CP_NoAd, by varying the number of requests from 50 to 300 , in a GT-ITM generated network with size 100, and real networks GÉANT, AS1755, and AS4755, respectively. It can be seen from Fig. 10 (a) that algorithms Online_Heu, SP, Online_Heu_NoAd can admit almost all requests if the number of requests is no greater 100. Otherwise, algorithm Online_Heu admits more requests than those by algorithms SP and Online_Heu_NoAd. Also, the performance gap between algorithms Online_Heu and SP increases with the growth of the number of requests. The reason is that online algorithm Online_Heu considers the workload (or the utilization) of each resource by assigning each resource an exponential cost, while algorithm SP does not take the resource workload and assigns the same weight to the same amount of resource at different nodes and links, which may lead to the excessive usage of a heavily-loaded resource. From Fig. 10 (e), we can also see the similar performance of algorithm Online_CP against algorithms Online_CP_NoAd and SP in GT-ITM generated topologies. Regarding their performance in networks GÉANT, AS1755, and AS4755, Figures 10 (b) - (d), and Figures 10 (f) - (h) illustrates similar trends of algorithms Online_Heu and Online_CP, respectively.

\subsection{Impact of parameters on the performance of differ- ent algorithms}

In the following we study the impact of the number of servers $M$ for implementing the service chain of each multicast on the performance of approximation algorithm Appro_Multi against that of approximation algorithm Alg_One_Server in real networks GÉANT, AS1755, and AS4755 [8], [38], by varying the value of $M$ from 2 to 5 . 


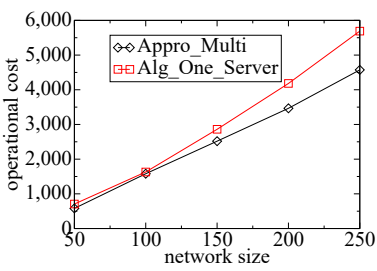

(a) Operational costs with $D_{\max } /|V|=0.05$

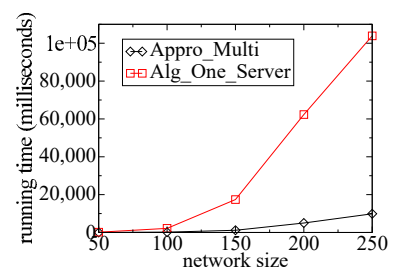

(d) Running times with $D_{\max } /|V|=0.05$

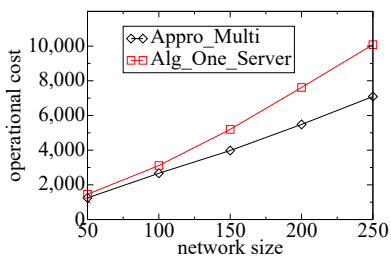

(b) Operational costs with $D_{\max } /|V|=0.1$

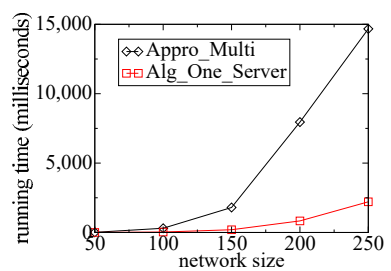

(e) Running times with $D_{\max } /|V|=0.1$

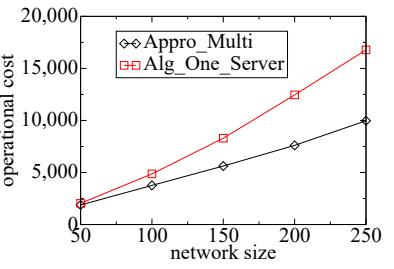

(c) Operational costs with $D_{\max } /|V|=0.2$

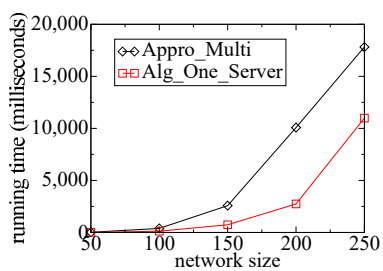

(f) Running times with $D_{\max } /|V|=0.2$

Fig. 6. The performance of algorithms Appro_Multi and Alg_One_Server with different ratios of $D_{\max } /|V|$.

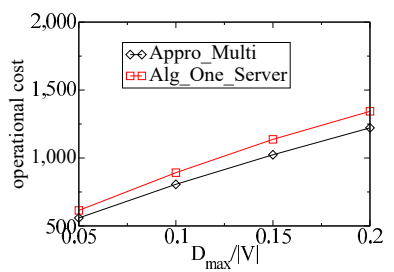

(a) Operational costs by different algorithms in GEANT

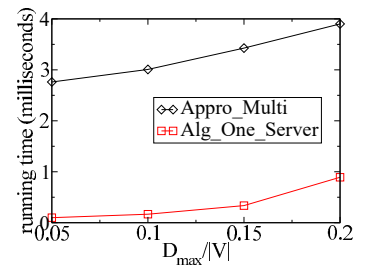

(c) Running times of different algorithms in GÉANT

Fig. 7. The performance of and AS1755 with $M=3$

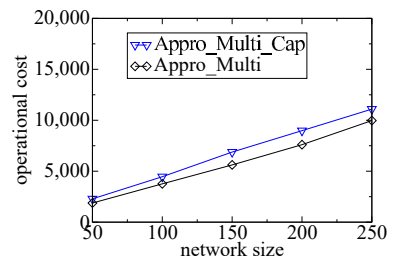

(a) The operational cost

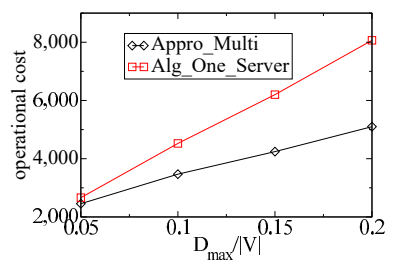

(b) Operational costs by different algorithms in AS1755

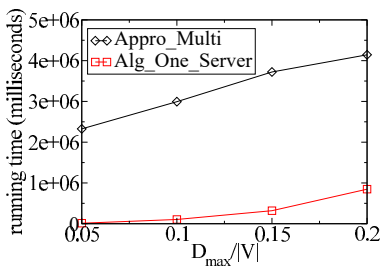

(d) Running times of different algorithms in AS1755
The results are depicted in Fig. 11. From Figures 11 (a), (b), and (c), it can be seen that the operational cost delivered by algorithm Appro_Multi is consistently lower than that by algorithm Alg_One_Server for different networks with different Ms. For example, as shown in Fig. 11 (c), the operational cost of approximation algorithm Appro_Multi in network AS4755 is at least 30\% less than that of algorithm Alg_One_Server. Also, the operational cost of algorithm

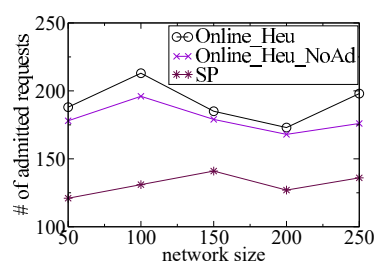

(a) The number of admitted requests

Fig. 9. The performance of online algorithms Online_Heu, Online_CP, and SP by varying the network sizes from 50 to 250 .

Appro_Multi decreases while its running time increases, with the increase of $M$, due to more server combinations to be explored.

We finally investigate the impact of $\alpha$ and $\beta$ on the performance of online algorithm Online_CP, by varying $\alpha$ and $\beta$ from $2^{1}|V|$ to $2^{5}|V|$ while setting $\sigma_{v}=\sigma_{e}=|V|-1$. Fig. 12 plots the performance curves of algorithm Online_CP. It can be seen from Fig. 12 (a) that when $\beta$ is fixed, the larger the value of $\alpha$, the less number of requests can be admitted by algorithm Online_CP. Also, when $\alpha$ is fixed, similar performance can be found in Fig. 12 (b).

\section{Conclusion}

In this paper we studied NFV-enabled multicasting in an SDN. We first devised an approximation algorithm with a constant approximation ratio, assuming that the number of servers for implementing each service chain is no more than a constant $M \geq 1$, subject to the computing and bandwidth capacity constraints. We then studied dynamic admissions of NFV-enabled multicast requests without the knowledge of future arrivals, with the objective to maximize the network throughput, for which we proposed an efficient heuristic and an online algorithm with a provable competitive ratio if $M=1$. We finally evaluated the performance of the proposed algorithms by experimental simulations. Simulation results demonstrate that the proposed algorithms outperform the other heuristics. 


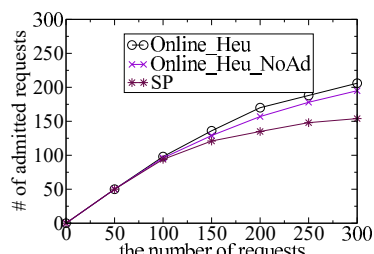

(a) The number of admitted requests by different algorithms in GT-ITM generated networks
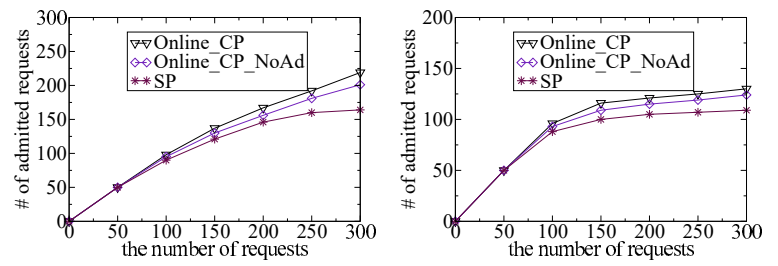

(e) The number of admit-(f) The number of admitted reted requests by different algo-quests by different algorithms rithms in GT-ITM generated in GÉANT networks

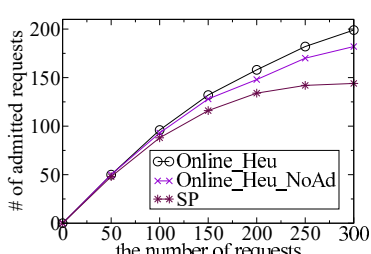

(b) The number of admitted requests by different algorithms in GÉANT

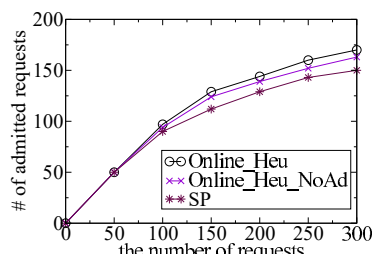

(c) The number of admitted requests by different algorithms in AS1755

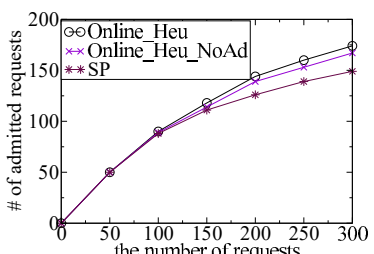

(d) The number of admitted requests by different algorithms in AS4755

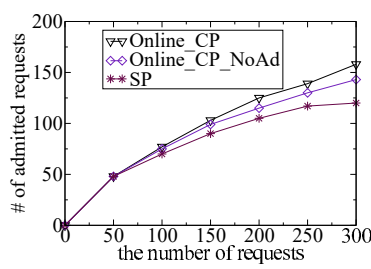

(g) The number of admitted requests by different algorithms in AS1755

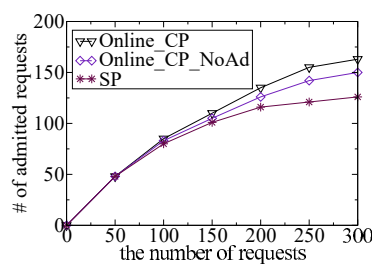

(h) The number of admitted requests by different algorithms in AS4755

Fig. 10. The performance of algorithms Online_Heu, Online_CP, and SP by varying the number of requests in a monitoring period from 50 to 300 while fixing the network size at 100 .

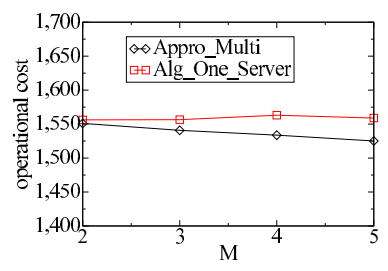

(a) Operational cost by differ-

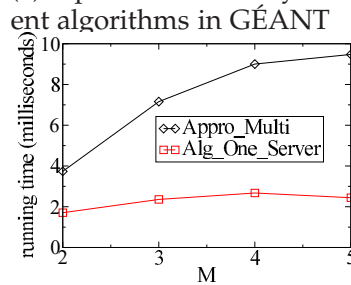

(d) Running times of different algorithms in GÉANT

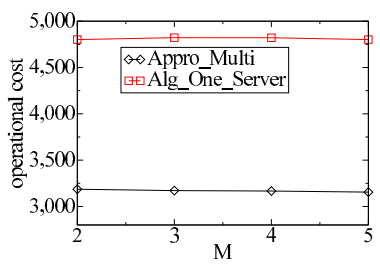

(b) Operational cost by different algorithms in AS1755

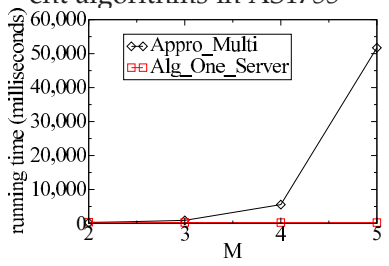

(e) Running times of different algorithms in AS1755

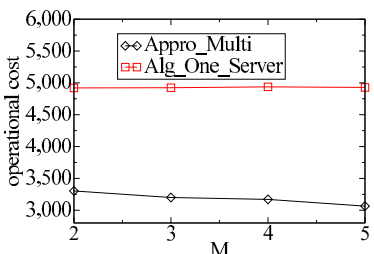

(c) Operational cost by different algorithms in AS4755

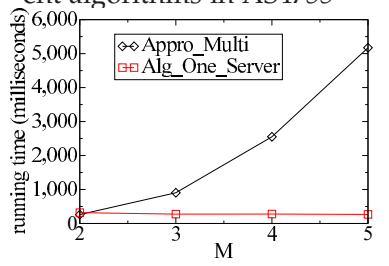

(f) Running times of different algorithms in AS4755

Fig. 11. The performance of algorithms Appro_Multi and Alg_one_Server in networks of GÉANT, AS1755, and AS4755.

(a) The number of admitted re-

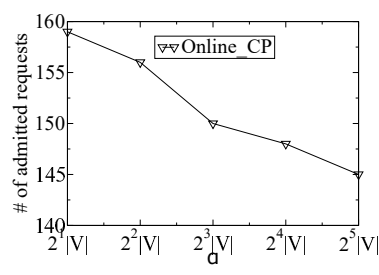
quests with $\beta=2^{1}|V|$

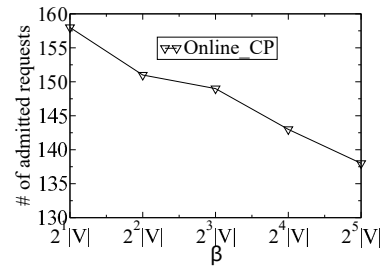

(b) The number of admitted requests with $\alpha=2^{1}|V|$
Fig. 12. The impact of parameters $\alpha$ and $\beta$ on the performance of algorithms Online_CP by varying $\alpha$ or $\beta$ while fixing the other at $2^{1}|V|$.

\section{ACKNOWLEDGEMENTS}

We would like to thank the three anonymous referees and the associate editor for their expertise comments and constructive suggestions, which have helped us improve the quality and presentation of the paper greatly. The work of
Zichuan $\mathrm{Xu}$ is supported by the National Natural Science Foundation of China (Grant No. 61802048), the fundamental research funds for the central universities in China (Grant No. DUT17RC(3)061), and the Xinghai Scholar Program in Dalian University of Technology, China. The work Alex Galis is partially supported by EU projects 5GEx (671636) and NECOS projects (777067).

\section{REFERENCES}

[1] Application Delivery Controller. http://searchnetworking.techtarget.com definition/Application-delivery-controller, 2017.

[2] J. Aspnes, Y. Azar, A. Fiat, S. Plotkin, and O. Waarts. On-line routing of virtual circuits with applications to load balancing and machine scheduling. J. ACM, vol. 44, pp.486-504, ACM, 1997.

[3] L. Barroso, J. Dean, and U. Holzle. Web search for a planet: The Google cluster architecture. IEEE Micro, vol. 23, no. 2, pp. 22-28, IEEE, 2003.

[4] J. Byrka, F. Grandoni, T. Rothvo, and L. Sanitá. An improved LPbased approximation for steiner tree. Proc. of STOC, ACM, 2010. 
[5] Z. Cao, M. Kodialam, and T. V. Lakshman. Traffic steering in software defined networks: planning and online routing. Proc. of DCC, ACM, 2014.

[6] R. Cohen, L. Eytan, J. Naor, and D. Raz. On the effect of forwarding table size on SDN network utilization. Proc. of INFOCOM, IEEE, 2014.

[7] R. Cohen, L. Eytan, J. Naor, and D. Raz. Near optimal placement of virtual network functions. Proc. of INFOCOM, IEEE, 2015.

[8] GÉANT. http://www.geant . net.

[9] S. Ghemawat, H. Gobioff, and S. Leung. The Goolge file system. Proc. of SOSP, 2003.

[10] http://www.cc.gatech.edu/projects/gtitm/.

[11] L. Guo, J. Pang, and A. Walid. Dynamic service function chaining in SDN-Enabled networks with middleboxes. Proc. of ICNP, IEEE, 2016

[12] A. Gushchin, A. Walid, and A. Tang. Scalable routing in SDNenabled networks with consolidated middleboxes. Proc. of HotMiddlebox, ACM, 2015.

[13] Hewlett-Packard Development Company. L.P. Servers for enterprise bladeSystem, rack \& tower and hyperscale. ttp:// www8.p.com/us/en/products/servers/, 2015.

[14] B. Han, V. Gopalakrishnan, L. Ji, and S. Lee. Network function virtualization: Challenges and opportunities for innovations. IEEE Communications Magazine, Vol. 53, No. 2, pp. 90 - 97, IEEE, 2015.

[15] L. Huang, H. Hung, C. Lin, and D. Yang. Scalable steiner tree for multicast communications in software-defined networking. Computing Research Repository (CoRR), vol. abs/1404.3454, 2014.

[16] M. Huang, W. Liang, Z. Xu, W. Xu, S. Guo and Y. Xu. Dynamic routing for network throughput maximization in software-defined networks. Proc. of INFOCOM, IEEE, 2016.

[17] K. Kar, M. Kodialam, T. V. Lakshman, and L. Tassiulas. Routing for network capacity maximization in energy-constraint ad-hoc networks. Prof. of INFOCOM, IEEE, 2003.

[18] D. Kreutz, F. M. V. Ramos, P. Esteves Verissimo, C. Esteve Rothenberg, S. Azodolmolky, and S. Uhlig. Software-Defined Networking: a comprehensive survey. Proceedings of the IEEE, Vol. 103, issue 1, pp. 14-76, IEEE, 2015.

[19] S. Knight et al. The internet topology zoo. J. Selected Areas in Communications, Vol. 29, pp. 1765 - 1775, IEEE, 2011.

[20] L. Kou, G. Markowsy, and L. Berman. A faster algorithm for Steiner trees. Acta Informatica, Volume 15, pp. 141-145, Springer, 1981.

[21] T-W. Kuo, B-H. Liou, K. C. Lin, and M-J Tsai. Deploying chains of virtual network functions: on the relation between link and server usage. Proc. of INFOCOM, IEEE, 2016.

[22] Y. Li, L. T. X. Phan, and B. T. Loo. Network functions virtualization with soft real-time guarantees. Proc. of INFOCOM, IEEE, 2016.

[23] W. Liang. Constructing minimum-energy broadcast trees in wireless ad hoc networks. Proc. of Mobihoc, ACM, 2002.

[24] W. Liang and X. Guo. On-line multicasting for network capacity maximization in energy-constrained ad hoc networks. IEEE Transactions on Mobile Computing, vol. 5, pp. 1215-1227, 2006.

[25] W. Liang and Y. Liu. On-line data gathering for maximizing network lifetime in sensor networks. IEEE Transactions on Mobile Computing, vol. 6, pp.2-11, 2007.

[26] T. Lukovszki and S. Schmid. Online admission control and embedding of service chains. Proc. of SIROCCO, 2015.

[27] L. Mamatas, S. Clayman, and A. Galis. Software-defined infrastructure. IEEE Communications Magazine, Vol. 53, No. 4, pp 166-174, IEEE, 2015.

[28] MapReduce. mapred_tutorial.html, 2017.

[29] J. Martins et al. ClickOS and the art of network function virtualization. Proc. of NSDI, USENIX, 2014.

[30] M. McBride. Multicast in the data center overview. IETF draft, https://tools.ietf.org/id/draft-ietf-mboned-dc-deploy00.html\#rfc.status, 2013.

[31] H. Moens and F. D. Turck. VNF-P: A model for efficient placement of virtualized network functions. Proc. of CNSM, IEEE, 2014.

[32] S. Plotkin. Competitive routing of virtual circuits in ATM networks. I. Selected Areas in Communications, IEEE, 1995

[33] Z. A. Qazi, C. C. Tu, L. Chiang, R. Miao, V. Sekar, M. Yu. SIMPLE-fying middlebox policy enforcement using SDN. Proc. of SIGCOMM, ACM, 2013.

[34] L. Qu, C. Assi, K. Shaban. Delay-aware scheduling and resource optimization with network function virtualization. IEEE Transactions on Communications, Vol. 64, No. 9, pp. 3746-3758, IEEE, 2016.
[35] G. Robins and A. Zelikovsky. Tighter bounds for graph steiner tree approximation. SIAM Journal on Discrete Mathematics, Vol. 19, No. 1, pp. 122-134, 2005.

[36] Software-defined data center. https://en.wikipedia.org/wiki/ Software-defined_data_center, accessed in 2017.

[37] Software-defined data center - In depth. https: / / www.vmware.com/solutions/software-defineddatacenter/in-depth.html, accessed in 2017.

[38] N. Spring, R. Mahajan, and D. Wetherall. Measuring ISP topologies with rocketfuel. Proc. of SIGCOMM, ACM, 2002.

[39] Z. Xu, W. Liang, A. Galis, and Y. Ma. Throughput maximization and resource optimization in NFV-enabled networks. Proc. of ICC, IEEE, 2017.

[40] Z. Xu, W. Liang, M. Huang, M. Jia, S. Guo, and A. Galis. Approximation and online algorithms for NFV-enabled multicasting in SDNs. Proc. of 37th Intl Conf on Distributed Computing Systems (ICDCS'17), IEEE, 2017.

[41] Y. Zhang et. al. StEERING: A software-defined networking for inline service chaining. Proc. of ICNP, IEEE, 2013.

[42] S. Q. Zhang, Q. Zhang, H. Bannazadeh, and A. L. Garcia. Network function virtualization enabled multicast routing on SDN. Proc. of ICC, IEEE, 2015

[43] S. Q. Zhang, Q. Zhang, H. Bannazadeh, and A. L. Garcia. Routing algorithms for network function virtualization enabled multicast topology on SDN. IEEE Transaction on Network and Service Management, Vol.12, No.4, pp.580-594, 2015.

\section{APPENDIX}

\section{Proof of Lemma 1}

Proof We here show that the solution delivered by Algorithm 1 is feasible. Each path $p$ in $T_{k}$ from $s_{k}^{\prime}$ to one destination $d \in D_{k}$ corresponds to a path in $G$ from $s_{k}$ to $d$. This is evidenced by the fact that any path in subgraph $H_{k}^{i}$ of $G_{k}^{i}$, starting from $s_{k}^{\prime}$ must use one of its incident edges in $E_{k}^{i}$, and the another endpoint $v$ of the edge must be a server in $V_{S}^{i} \subseteq V_{S}$, following the construction of $G_{k}^{i}$. This implies that each path in $T_{k}$ from $s_{k}$ to any destination $d \in D_{k}$ must use one of the servers in $V_{S}^{i}$. And $T_{k}$ includes all nodes in $D_{k}$. Thus, the tree obtained is a feasible pseudo-multicast tree for multicast request $r_{k}$.

\section{Proof of Lemma 2}

Proof Following the construction of $G_{k}^{i}$, each approximate Steiner tree $T_{k}^{i}$ in $G_{k}^{i}$ will include at least one edge between the virtual source node $s_{k}^{\prime}$ and a switch node attached to server $v_{j} \in V_{S}^{i}$, which is replaced by the corresponding shortest path $p_{s_{k}, v_{j}}$ in $G$ between $s_{k}$ and $v_{j}$ for the admission of request $r_{k}$. However, there may exist an edge $e \in E$ which is in both $T_{k}^{i} \backslash\left\{\left(s_{k}^{\prime}, v_{j}\right) \mid v_{j} \in V_{S}^{i}\right\}$ and $p_{s_{k}, v_{j}}$. Whenever this happens, edge $e$ will be used twice in the data traffic routing of request $r_{k}$ : one is when $e$ is in path $p_{s_{k}, u}$ between the source $s_{k}$ and a server $u \in V_{S}^{i}$, where the edge $\left(s_{k}^{\prime}, u\right) \in\left\{\left(s_{k}^{\prime}, v_{j}\right) \mid v_{j} \in V_{S}^{i}\right\} \subset E_{k}^{i}$ derived from server $u$ is included in $T_{k}^{\text {min }}$; and another is that edge $e$ is in a path between server $u$ and at least one destination in $D_{k}$. Thus, each link $e \in E_{k}^{i}$ must have at least the amount $2 b_{k}$ of residual bandwidth for the admission of request $r_{k}$, to ensure that the data traffic of request $r_{k}$ can be routed from its source $s_{k}$ to each of its destinations.

\section{Proof of Theorem 1}

Proof We first analyze the approximation ratio of Algorithm 1 . Let $G_{T}^{*}$ be the optimal pseudo-multicast tree for the NFV-enabled multicast request $r_{k}$ in $G$. If $G_{T}^{*}$ is not a multicast tree, there is a corresponding tree $T^{\prime}$ 
with the identical cost as $G_{T}^{*}$, following the reduction in subsection 3.2; otherwise, $G_{T}$ itself is a multicast tree. From now on, we denote by $T^{*}$ either the optimal multicast tree $G_{T}^{*}$ or its corresponding cost-identical tree $T^{\prime}$. We assume that there are $l$ servers in $T^{*}$ with each implementing $S C_{k}$ with $1 \leq l \leq M$. Without loss of generality, we assume that these $l$ nodes are $v_{1}, v_{2}, \ldots, v_{l}$, respectively. Clearly, it can be shown that none of pairs of these nodes in $T^{*}$ has the ancestor and descendant relationship in terms of a node being used as a server, otherwise the node in $V_{S}$ will be treated as a regular switch node without the use of its server. Each subtree $T_{v_{i}}^{*}$ of $T^{*}$ rooted at $v_{i}$ contains some destinations, and all of the $l$ subtrees will contain all the destinations in $D_{k}$, following its definition. We construct another tree $T_{c}^{*}=\left(V^{\prime}, E^{\prime}\right)$ which is derived from $T^{*}$ by compressing the path in $T^{*}$ from $s_{k}$ to each node $v_{i}$ as follows. We replace the source node $s_{k}$ by a node $s_{k}^{\prime}$ and the path in $T^{*}$ from $s_{k}$ to $v_{i}$ by an edge $\left(s_{k}^{\prime}, v_{i}\right)$, and assign the edge a weight that is the sum of all edge costs in the path plus the cost of using server $v_{i}$. In the worst case, each of such compressions can increase the cost of the optimal tree, and there are in total $l$ compressions. Furthermore, for each compression, if the cost sum of all edges from the source $s_{k}$ to each $v_{i}$ dominates the cost of the tree, each of such compressions can increase the total cost by a value that equals the cost of $T^{*}$. We thus claim that the cost of tree $T_{c}^{*}$ is no greater than $l$ times the cost of tree $T^{*}$, i.e., $c\left(T_{c}^{*}\right) \leq l \cdot c\left(T^{*}\right)$.

It can be seen that there is a multicast tree $T_{k}^{i}$ in $G_{k}^{i}$ rooted at source $s_{k}^{\prime}$ and spanning all destinations in $D_{k}$, which has the same topological structure as $T_{c}^{*}$, however, it has a lower cost compared with that of $T_{c}^{*}$, i.e., $c\left(T_{k}^{i}\right) \leq c\left(T_{c}^{*}\right)$. This is because the weight of each edge in $G_{k}^{i}$ between $s_{k}^{\prime}$ and $v_{i}$ is the length of the shortest path in $G$ between the two nodes plus the cost of using server $v_{i}$, while the corresponding edge weight in $T_{c}^{*}$ is the sum of all edge weights in the path in $T^{*}$ between $s_{k}$ and $v_{i}$ plus the cost of using server $v_{i}$.

Let $T_{k}^{O P T, i}$ be an optimal multicast tree in $G_{k}^{i}$ rooted at $s_{k}^{\prime}$ and spanning all destinations in $D_{k}$ and each path in the tree from $s_{k}^{\prime}$ to a destination goes through one of the servers in $V_{S}^{\prime}$. Then, $c\left(T_{k}^{O P T, i}\right) \leq c\left(T_{k}^{i}\right)$ as $T_{k}^{i}$ is one of the multicast trees for multicast request $r_{k}$. Let $T_{k}^{a p p, i}$ be an approximate multicast tree in $G_{k}^{i}$ for multicast request $r_{k}$, by the approximation algorithm with an approximation ratio of 2 due to Kou et al. [20]. We then have $c\left(T_{k}^{a p p, i}\right) \leq$ $2 c\left(T_{k}^{O P T, i}\right)$. Since $c\left(T_{k}^{i}\right) \leq c\left(T_{c}^{*}\right)$ and $c\left(T_{c}^{*}\right) \leq l \cdot c\left(T^{*}\right)$, we have $c\left(T_{k}^{a p p, i}\right) \leq 2 c\left(T_{k}^{O P T, i}\right) \leq 2 c\left(T_{k}^{i}\right) \leq 2 c\left(T_{c}^{*}\right) \leq 2 \cdot l$. $c\left(T^{*}\right)$. Since a pseudo-multicast tree $T_{k}$ with the minimum

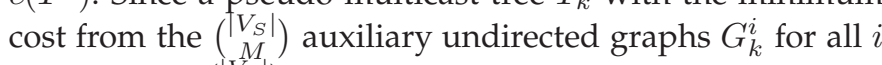
with $1 \leq i \leq\left(\begin{array}{c}\left|V_{S}\right| \\ M\end{array}\right)$ will be found and the value of $l$ is within $[1, M]$, the cost of the pseudo-multicast tree $T_{k}$ for $r_{k}$ is no greater than $2 M \cdot c\left(T^{*}\right)$.

We finally analyze the time complexity of Algorithm 1 as follows. The algorithm proceeds iteratively. Within each iteration, it first constructs an auxiliary graph $G_{k}^{i}$, and then finds an approximate Steiner tree $T_{k}^{a p p, i}$ in $G_{k}^{i}$ for each multicast request $r_{k}$. It takes $O(|E|+|V| \log |V|)$ time to find a single-source shortest path tree in $G_{k}$ by Dijkstra's algorithm, while it takes $O\left(\left|V_{k}^{i}\right|^{3}\right)=O\left(|V|^{3}\right)$ time to find an approximate Steiner tree $T_{k}^{a p p, i}$ [20]. There are $O\left(\left(\begin{array}{c}\left|V_{S}\right| \\ M\end{array}\right)\right)\left(=O\left(\left|V_{S}\right|^{M}\right)\right)$ iterations. The algorithm thus takes $O\left(|V|^{3} \cdot\left|V_{S}\right|^{M}\right)$ time. For example, if $\left|V_{S}\right|=O(\log |V|)$ and $M=3$, then the time complexity of Algorithm 1 is $O\left(|V|^{3} \cdot \log ^{3}|V|\right)$.

\section{Proof of Theorem 2}

Proof We first show that the solution delivered by Algorithm 2 is feasible, meeting the computing and bandwidth resource demands of multicast request $r_{k}$. This can be evidenced by the construction of $G_{k}^{i}$ for all $i$ with $1 \leq i \leq\left(\begin{array}{c}\left|V_{S}(k)\right| \\ M\end{array}\right)$. Specifically, for meeting the computing resource demands of request $r_{k}$, only the switch nodes whose attached servers with sufficient available computing resource will be included in $V_{S}^{i}$. For the bandwidth resource demand $b_{k}$ of multicast request $r_{k}$, an amount $b_{k}$ of bandwidth resource is reserved on each link in the shortest path $p_{s_{k}, v}$ in $G$ from $s_{k}$ to each $v \in V_{S}$ for the transfer of the original traffic of $r_{k}$, and an equal amount is allocated to transfer the processed traffic by each selected server to destinations in $D_{k}$. By Lemma 2, it can be seen that the bandwidth capacity constraint on each link is met. Notice that if a switch $v^{\prime} \in V_{S}$ is not selected in the solution, the preserved resource $b_{k}$ in each edge of path $p_{s_{k}, v}$ will be released to the resource pool. Also, following the construction of $G_{k}^{i}$, it can be seen that each routing path from $s_{k}^{\prime}$ to a destination node $u$ must pass through a node $u$ in the path and $u$ is the neighbor of $s_{k}^{\prime}, u \in V_{S}^{i}$ is a server with sufficient computing resource for $S C_{k}$, i.e., the available computing resource at $u$ is at least $C_{u}\left(S C_{k}\right)$. Thus, the solution is a feasible solution.

We then analyze the running time of the proposed online algorithm. Clearly, the construction of auxiliary graph $G_{k}^{i}$ takes $O(|V| \log |V|+|E|)$ time, as its edge assignment needs finding a single-source shortest path tree in $G$ which takes $O(|V| \log |V|+|E|)$ time. Finding an approximate Steiner tree in $G_{k}^{i}$ takes $O\left(\left|V_{k}^{i}\right|^{3}\right)=O\left(|V|^{3}\right)$ time, by the approximation algorithm in [20]. There are $O\left(\left(\begin{array}{c}\left|V_{S}(k)\right| \\ M\end{array}\right)\right)(=$ $\left.O\left(\left|V_{S}(k)\right|^{M}\right)=O\left(\left|V_{S}\right|^{M}\right)\right)$ different values of $i$, and transforming a minimum-cost approximate Steiner tree $T_{k}^{\min }$ to a feasible solution of the problem takes $O\left(\left|V_{S}\right|+|V|\right)$ time. Therefore it takes $O\left(\left(|V| \log |V|+|E|+|V|^{3}\right) \cdot\left|V_{S}\right|^{M}\right)=$ $O\left(|V|^{3} \cdot\left|V_{S}\right|^{M}\right)$ time for each incoming multicast request. The algorithm thus takes $O\left(k|V|^{3}\left|V_{S}\right|^{M}\right)$ time if there are $k$ NFV-enabled multicast requests in the request sequence. The theorem follows.

\section{Proof of Lemma 3}

Proof Consider any $k^{\prime} \in \mathcal{S}(k)$ admitted by the online algorithm, its data traffic is first routed to a server $v \in V_{S}$ that hosts its service chain $S C_{k}$ via path $p_{s_{k}, v}$ in $G$, and then to each of its destinations in $D_{k}$ through a subtree $T_{v}$ with root $v$. The costs of computing resource usage of different servers in $V_{S}$ are different, we thus have

$$
\begin{aligned}
& c_{v}\left(k^{\prime}+1\right)-c_{v}\left(k^{\prime}\right) \leq C_{v}\left(\alpha^{1-\frac{C_{v}\left(k^{\prime}+1\right)}{C_{v}}}-\alpha^{1-\frac{C_{v}\left(k^{\prime}\right)}{C_{v}}}\right) \\
& =C_{v} \alpha^{1-\frac{C_{v}\left(k^{\prime}\right)}{C_{v}}}\left(\alpha^{\frac{C_{v}\left(S C_{k^{\prime}}\right)}{C_{v}}}-1\right) \\
& =C_{v} \alpha^{1-\frac{C_{v}\left(k^{\prime}\right)}{C_{v}}}\left(2^{\frac{C_{v}\left(S C_{k^{\prime}}\right) \log \alpha}{C_{v}}}-1\right)
\end{aligned}
$$




$$
\begin{aligned}
& \leq C_{v} \alpha^{1-\frac{C_{v}\left(k^{\prime}\right)}{C_{v}}}\left(\frac{C_{v}\left(S C_{k^{\prime}}\right) \log \alpha}{C_{v}}\right) \\
& =\alpha^{1-\frac{C_{v}\left(k^{\prime}\right)}{C_{v}}} C_{v}\left(S C_{k^{\prime}}\right) \cdot \log \alpha .
\end{aligned}
$$

Notice that the derivation from Eq. (7) to inequality (8) is due to that $2^{x}-1 \leq x$ for $0 \leq x \leq 1$. Similarly, if edge $e \in E$ is in a pseudo-multicast tree for multicast request $r_{k}$, we have

$$
c_{e}\left(k^{\prime}+1\right)-c_{e}\left(k^{\prime}\right) \leq \beta^{1-\frac{B_{e}\left(k^{\prime}\right)}{B_{e}}} b_{k^{\prime}} \cdot \log \beta .
$$

Notice that $c_{v}(0)=0$ for all $v \in V_{S}$. If an NFV-enabled multicast request $r_{k^{\prime}}$ is admitted, this means that

$$
w_{v}\left(k^{\prime}\right)=\alpha^{1-\frac{C_{v}\left(k^{\prime}\right)}{C_{v}}}-1 \leq \sigma_{v}=|V|-1,
$$

We now calculate the cost sum of all edges and the server attached to $v \in V_{S}$ when admitting the multicast request $r_{k}$. Notice that if an edge in $E$ is not included in $T_{k}$ for $r_{k}$, its cost does not change after the admission of $r_{k}$. So does the cost of a server that is not used for implementing $S C_{k}$ of $r_{k}$. Assuming that $v$ is chosen for $r_{k^{\prime}}$, we have

$\sum_{v \in V_{S}} c_{v}\left(k^{\prime}+1\right)-c_{v}\left(k^{\prime}\right)=c_{v}\left(k^{\prime}+1\right)-c_{v}\left(k^{\prime}\right)$

$\leq\left(\alpha^{1-\frac{C_{v}\left(k^{\prime}\right)}{C_{v}}}\right) C_{v}\left(S C_{k^{\prime}}\right) \log \alpha$, by inequality (9)

$=\left(\alpha^{1-\frac{C_{v}\left(k^{\prime}\right)}{C_{v}}}-1\right) C_{v}\left(S C_{k^{\prime}}\right) \log \alpha+C_{v}\left(S C_{k^{\prime}}\right) \log \alpha$

$\leq\left(\alpha^{1-\frac{C_{v}\left(k^{\prime}\right)}{C_{v}}}-1\right) C_{v}\left(S C_{k^{\prime}}\right) \log \alpha+(|V|-1) C_{v}\left(S C_{k^{\prime}}\right) \log \alpha$ since $|V|>1$

$\leq 2 C_{v}\left(S C_{k^{\prime}}\right) \log \alpha(|V|-1)$ by inequality (11).

Similarly we have

$$
\sum_{e \in E} c_{e}\left(k^{\prime}+1\right)-c_{e}\left(k^{\prime}\right) \leq 2 b_{k^{\prime}} \log \beta(|V|-1) .
$$

We finally have

$$
\begin{aligned}
& \sum_{v \in V_{S}} c_{v}(k)=\sum_{k^{\prime}=1}^{k-1} \sum_{v \in V_{S}}\left(c_{v}\left(k^{\prime}+1\right)-c_{v}\left(k^{\prime}\right)\right) \\
& \leq \sum_{k^{\prime} \in \mathcal{S}(k)} 2 C_{v}\left(S C_{k^{\prime}}\right) \cdot \log \alpha \cdot(|V|-1) \\
& =2 \mathbb{C}(k) \cdot \log \alpha \cdot(|V|-1),
\end{aligned}
$$

and similarly $\sum_{e \in E} c_{e}(k) \leq 2 \mathbb{B}(k) \cdot \log \beta \cdot(|V|-1)$.

\section{Proof of Lemma 4}

Proof A multicast request $r_{k^{\prime}}$ will be rejected by the proposed online algorithm, Algorithm 3, because of the following cases. Case 1, there is no sufficient computing resource for implementing the service chain of $r_{k^{\prime}}$; Case 2. There is no sufficient bandwidth resource for routing the traffic of $r_{k^{\prime}}$ to its destinations; Case 3. The weighted sum of edges in the pseudo-multicast tree for $r_{k^{\prime}}$ is too high (Step 9), and/or the weight of the selected server attached to switch $v$ to implement the service chain of $r_{k^{\prime}}$ is too high (Step 7).

Case 1 . Let $v^{\prime}$ be the switch whose attached server is selected to implement the service chain of request $k^{\prime}$. As the request is rejected by Algorithm 3 due to insufficient available computing resource, we have $C_{v^{\prime}}\left(k^{\prime}\right)<C_{v}\left(k^{\prime}\right)$. Therefore, $1-\frac{C_{v^{\prime}}\left(k^{\prime}\right)}{C_{v^{\prime}}} \geq 1-\frac{C_{v}\left(k^{\prime}\right)}{C_{v}\left(S C_{k^{\prime}}\right)} \geq 1-\frac{1}{\log \alpha}$, since
$C_{v}\left(S C_{k}\right) \leq \frac{\min _{v \in V_{S}} C_{v}}{\log \alpha}$. We then have

$$
\begin{gathered}
w\left(T_{k^{\prime}}^{\prime}\right)+w_{v^{\prime}}\left(k^{\prime}\right) \geq \alpha^{1-\frac{C_{v^{\prime}\left(k^{\prime}\right)}}{C_{v^{\prime}}}}-1>\alpha^{1-\frac{1}{\log \alpha}}-1 \\
=\frac{\alpha}{2}-1=|V|-1 .
\end{gathered}
$$

Case 2. If request $r_{k^{\prime}}$ is rejected in this case, there exists an edge $e^{\prime} \in T_{k^{\prime}}^{\prime}$ that does not have enough bandwidth resource. This implies that for edge $e^{\prime}$, we have $B_{e^{\prime}}\left(k^{\prime}\right)<$ $b_{k^{\prime}}$. Thus, we have $1-\frac{B_{e^{\prime}}\left(k^{\prime}\right)}{B_{e^{\prime}}} \leq 1-\frac{b_{k^{\prime}}}{B_{e^{\prime}}} \leq 1-\frac{1}{\log \beta}$, since $b_{k^{\prime}} \leq \frac{\min _{e \in E} B_{e}}{\log \beta}$. Similarly, we have

$$
\begin{aligned}
& w\left(T_{k^{\prime}}^{\prime}\right)+w_{v^{\prime}}\left(k^{\prime}\right) \geq \sum_{e \in T_{k^{\prime}}^{\prime}} \beta^{1-\frac{B_{e}\left(k^{\prime}\right)}{B_{e}}}-1 \\
& \geq \beta^{1-\frac{B_{e^{\prime}\left(k^{\prime}\right)}}{B_{e^{\prime}}}}-1 \geq \beta^{1-\frac{1}{\log \beta}}-1 \geq \frac{\beta}{2}-1=|V|-1 .
\end{aligned}
$$

Case 3. Let $T_{k^{\prime}}$ be the pseudo-multicast tree by Algorithm 3 for multicast request $r_{k^{\prime}}$. According to inequality (5), we have $w\left(T_{k^{\prime}}\right)+w_{v^{\prime}}\left(k^{\prime}\right) \leq 4\left(w\left(T_{k^{\prime}}^{*}\right)+\right.$ $\left.w_{v^{\prime}}\left(k^{\prime}\right)\right) \leq 4\left(w\left(T_{k^{\prime}}^{\prime}\right)+w_{v^{\prime}}\left(k^{\prime}\right)\right)$, where $T_{k^{\prime}}^{*}$ and $v^{\prime}$ are the optimal multicast tree and the server for $r_{k^{\prime}}$ by the optimal solution. Since the proposed online algorithm rejected the request, we have $w\left(T_{k^{\prime}}\right)>\sigma_{e}$ and/or $w_{v^{\prime}}\left(k^{\prime}\right)>\sigma_{v}$. If $w\left(T_{k^{\prime}}\right)>\sigma_{e}$, we have $w\left(T_{k^{\prime}}^{\prime}\right)+w_{v^{\prime}}\left(k^{\prime}\right) \geq \frac{w\left(T_{k^{\prime}}\right)+w_{v^{\prime}}\left(k^{\prime}\right)}{4} \geq$ $\frac{w\left(T_{k^{\prime}}\right)}{4} \geq \frac{\sigma_{e}}{4}=\frac{|V|-1}{4}$, similarly, if $w_{v^{\prime}}\left(k^{\prime}\right)>\sigma_{v}$, we have $w\left(T_{k^{\prime}}^{\prime}\right)+w_{v^{\prime}}\left(k^{\prime}\right) \geq \frac{\sigma_{v}}{4}=\frac{|V|-1}{4}$. Summarizing the three cases, we have $w\left(T_{k^{\prime}}^{\prime}\right)+w_{v^{\prime}}\left(k^{\prime}\right) \geq \frac{|V|-1}{4}$.

\section{Proof of Theorem 3}

Proof The competitive ratio of Algorithm 3 is analyzed as follows. Let $T_{k^{\prime}}^{*}$ be the optimal multicast tree by the optimal offline algorithm for request $r_{k^{\prime}} \in \mathcal{R}(k)$ and $v^{*} \in T_{k^{\prime}}^{*}$ be the server for the service chain $S C_{k^{\prime}}$ of $r_{k^{\prime}}$.

$$
\begin{aligned}
& \frac{|V|-1}{4}(|\mathcal{R}(k)|) \leq \sum_{r_{k^{\prime}} \in \mathcal{R}(k)} \frac{|V|-1}{4} \\
& \leq \sum_{r_{k^{\prime}} \in \mathcal{R}(k)} \sum_{e \in T_{k^{\prime}}^{*}}\left(\beta^{1-\frac{B_{e}\left(k^{\prime}\right)}{B_{e}}}-1\right)+\alpha^{1-\frac{C_{v^{*}\left(k^{\prime}\right)}}{C_{v^{*}}}}-1, \text { by Lemma } 4 \\
& =\sum_{r_{k^{\prime}} \in \mathcal{R}(k)}\left(\sum_{e \in T_{k^{\prime}}^{*}} c_{e}\left(k^{\prime}\right) / B_{e}+c_{v^{*}}\left(k^{\prime}\right) / C_{v^{*}}\right) \\
& \leq \sum_{r_{k^{\prime}} \in \mathcal{R}(k)}\left(\sum_{e \in T_{k^{\prime}}^{*}} c_{e}(k) / B_{e}+c_{v^{*}}(k) / C_{v^{*}}\right) \\
& \leq \sum_{e \in T_{k^{\prime}}^{*}} c_{e}(k) \sum_{r_{k^{\prime}} \in \mathcal{R}(k)} 1 / B_{e}+c_{v^{*}}(k) \sum_{r_{k^{\prime}} \in \mathcal{R}(k)} 1 / C_{v^{*}} \\
& \leq \sum_{e \in E} c_{e}(k)+\sum_{v \in V_{S}} c_{v}(k) .
\end{aligned}
$$

Following inequalities (16) and Lemma 3, we have

$$
\begin{aligned}
& \frac{|V|-1}{4}(|\mathcal{R}(k)|)<\sum_{e \in E} c_{e}(k)+\sum_{v \in V_{S}} c_{v}(k) \\
& \leq 2 \mathbb{C}(k) \log \alpha(|V|-1)+2 \mathbb{B}(k) \log \beta(|V|-1) \\
& \leq 2|\mathcal{S}(k)| C_{\max } \log \alpha(|V|-1)+2|\mathcal{S}(k)| b_{\max } \log \beta(|V|-1) \\
& =2|\mathcal{S}(k)|(|V|-1)\left(C_{\max } \log \alpha+b_{\max } \log \beta\right),
\end{aligned}
$$

where $C_{\max }=\arg \max _{k} C_{v}\left(S C_{k}\right)$ and $b_{\max }=\arg \max _{k} b_{k}$, i.e., the maximum computing and bandwidth resource de- 
mands of all requests. Inequality (17) then can be rewritten as

$$
|\mathcal{R}(k)| /|\mathcal{S}(k)| \leq 8\left(C_{\max } \log \alpha+b_{\max } \log \beta\right) .
$$

The competitive ratio of Algorithm 3 thus is

$$
\begin{aligned}
& \frac{|\mathcal{S}(k)|}{|O P T|} \geq \frac{|\mathcal{S}(k)|}{|\mathcal{R}(k) \cup \mathcal{S}(k)|} \\
& \geq \frac{|\mathcal{S}(k)|}{|\mathcal{R}(k)|+|\mathcal{S}(k)|}=\frac{1}{|\mathcal{R}(k)| /|\mathcal{S}(k)|+1} \\
& \geq \frac{1}{1+8\left(C_{\max } \log \alpha+b_{\max } \log \beta\right)}, \text { by inequality (18) } \\
& \geq \frac{1}{c^{\prime} \log |V|}, \text { when } C_{\max } \text { and } b_{\max } \text { are given constants, }
\end{aligned}
$$

where $c^{\prime}>0$ is a positive constant and $\alpha=\beta=2|V|$. The competitive ratio of the competition ratio of Algorithm 3 thus is $O(\log |V|)$ when $\alpha=\beta=2|V|$.

The rest is to analyze the time complexity of the proposed algorithm. The construction of $G_{k}$ takes $O\left(\left|V_{k}\right|+\right.$ $\left.\left|E_{k}\right|\right)=O(|V|+|E|)$ time, while finding an approximate multicast tree from $G_{k}$ takes $O\left(\left|V_{k}\right|^{3}\right)=O\left(|V|^{3}\right)$ time, by the 2-approximation algorithm of Kou et al. [20]. Algorithm 3 thus takes $O\left(k|V|^{3}\right)$ time if there are $k$ requests.

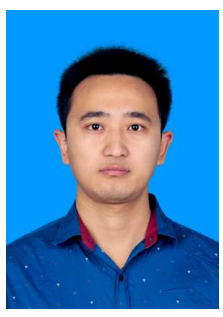

Zichuan Xu (M'17) received his $\mathrm{PhD}$ degree from the Australian National University in 2016, ME degree and BSc degree from Dalian University of Technology in China in 2011 and 2008, all in Computer Science. He is currently an Associate Professor at School of Software, Dalian University of Technology, China. He was a Research Associate at Department of Electronic and Electrical Engineering, University College London, UK. His research interests include cloud computing, network function virtualization, software-defined networking, wireless sensor networks, routing protocol design for wireless networks, algorithmic game theory, and optimization problems.

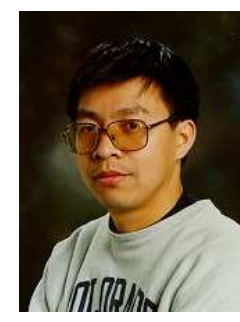

Weifa Liang (M'99-SM'01) received the PhD degree from the Australian National University in 1998, the ME degree from the University of Science and Technology of China in 1989, and the BSc degree from Wuhan University, China in 1984, all in computer science. He is currently a Full Professor in the Research School of Computer Science at the Australian National University. His research interests include design and analysis of energy efficient routing protocols for wireless ad hoc and sensor networks, cloud computing, Software-Defined Networking, design and analysis of parallel and distributed algorithms, approximation algorithms, combinatorial optimization, and graph theory. He is a senior member of the IEEE and a member of the ACM.

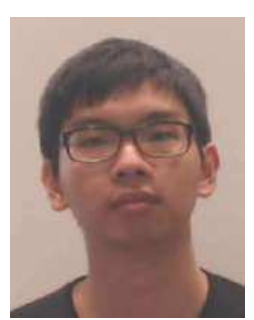

Meitian Huang received the BSc degree with the first class Honours in Computer Science at the Australian National University in 2015. He currently is studying for his PhD degree in the Research School of Computer Science at the Australian National University. His research interests include software-defined networking, algorithm design and analysis, and cloud computing.

Mike Jia is a second year PhD student in Computer Science at the Australian National University. His research interests include mobile cloud computing and software defined networks. He received the BSc degree in Mathematics and Computer Science from Imperial College London in UK in 2013, and an Honours in Computer Science at the Australian National University (2014).

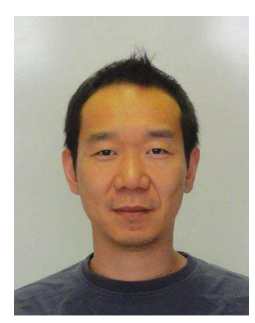

Song Guo (M'02-SM'11) received the PhD degree in computer science from the University of Ottawa, Canada in 2005. He is currently a Full Professor at the Department of Computing, the Hong Kong Polytechnic University, Hong Kong. His research interests are mainly in the areas of protocol design and performance analysis for wireless networks and distributed systems. He has published over 250 papers in refereed journals and conferences in these areas and received three IEEE/ACM best paper awards. Dr. Guo currently serves as Associate Editor of IEEE Transactions on Parallel and Distributed Systems, Associate Editor of IEEE Transactions on Emerging Topics in Computing for the track of Computational Networks, and on editorial boards of many others. He has also been in organizing and technical committees of numerous international conferences. Dr. Guo is a senior member of the IEEE and the ACM.

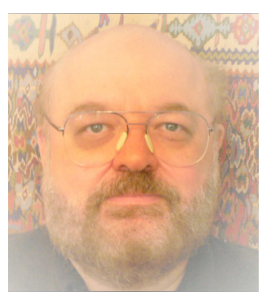

Alex Galis is a Professor in Networked and Service Systems at University College London. $\mathrm{He}$ has co-authored 10 research books and more that 250 publications in the Future Internet areas: system management, networks and services, networking clouds, $5 \mathrm{G}$ virtualisation and programmability. He was a member of the Steering Group of the Future Internet Assembly (FIA) and he led the Management and Service-aware Networking Architecture (MANA) working group. He acted as TPC chair of 14 IEEE conferences. $\mathrm{He}$ is also a co-editor of the IEEE Communications Magazine feature topic on Advances In Networking Software. He acted as a Vice Chair of the ITU-T SG13 Group on Future Networking. He is involved in IETF and ITU-T SG13 network slicing activities and he is also involved in IEEE SDN initiative. 